\title{
The neurotranscriptome of the Aedes aegypti mosquito
}

Benjamin J. Matthews ${ }^{1}$, Carolyn S. McBride ${ }^{1,3}$, Matthew DeGennaro ${ }^{1,4}$, Orion Despo ${ }^{1,5}$ and Leslie B. Vosshall ${ }^{1,2^{*}}$

\begin{abstract}
Background: A complete genome sequence and the advent of genome editing open up non-traditional model organisms to mechanistic genetic studies. The mosquito Aedes aegypti is an important vector of infectious diseases such as dengue, chikungunya, and yellow fever and has a large and complex genome, which has slowed annotation efforts. We used comprehensive transcriptomic analysis of adult gene expression to improve the genome annotation and to provide a detailed tissue-specific catalogue of neural gene expression at different adult behavioral states.

Results: We carried out deep RNA sequencing across all major peripheral male and female sensory tissues, the brain and (female) ovary. Furthermore, we examined gene expression across three important phases of the female reproductive cycle, a remarkable example of behavioral switching in which a female mosquito alternates between obtaining blood-meals from humans and laying eggs. Using genome-guided alignments and de novo transcriptome assembly, our re-annotation includes 572 new putative protein-coding genes and updates to 13.5 and $50.3 \%$ of existing transcripts within coding sequences and untranslated regions, respectively. Using this updated annotation, we detail gene expression in each tissue, identifying large numbers of transcripts regulated by blood-feeding and sexually dimorphic transcripts that may provide clues to the biology of male- and female-specific behaviors, such as mating and blood-feeding, which are areas of intensive study for those interested in vector control.

Conclusions: This neurotranscriptome forms a strong foundation for the study of genes in the mosquito nervous system and investigation of sensory-driven behaviors and their regulation. Furthermore, understanding the molecular genetic basis of mosquito chemosensory behavior has important implications for vector control.
\end{abstract}

Keywords: Mosquito, Aedes aegypti, mRNA-sequencing, De novo genome assembly, Host-seeking behavior, Neural genes, Chemosensory receptors, Ion channels, G protein-coupled receptors, Gonotrophic cycle, Neurogenetics

\section{Background}

Studies in classic genetic model organisms including the mouse, zebrafish, fly, worm and yeast have led to major advances in biology. All of these systems have in common a sequenced genome and the ability to carry out forward and reverse genetic manipulations. Non-model organisms, such as the mosquitoes we study, have not been accessible to mechanistic genetic studies until recently. The availability of genomes, next-generation sequencing and genome editing technologies now make it possible to apply modern genetics to study animals with

\footnotetext{
* Correspondence: Leslie.Vosshall@rockefeller.edu

'Laboratory of Neurogenetics and Behavior, The Rockefeller University, New York, NY 10065, USA

${ }^{2}$ Howard Hughes Medical Institute, New York, NY 10065, USA

Full list of author information is available at the end of the article
}

important and interesting biology previously inaccessible to molecular genetics.

Aedes aegypti is the primary vector for dengue, chikungunya and yellow fever - debilitating diseases that together are responsible for hundreds of millions of infections and thousands of deaths annually worldwide [1]. Female mosquitoes exhibit remarkable behavioral shifts throughout their adult life. Ae. aegypti are generally anautogenous, meaning that they do not produce eggs without a blood-meal [2]. Female Ae. aegypti use a variety of chemical and physical cues to locate hosts in their environment and to discriminate humans from non-human animals [3-8]. Although male Ae. aegypti do not feed on blood, they also respond to host chemosensory cues, perhaps to locate females congregating near humans [9]. At short range, the male locates a potential mate using 
the specific frequencies generated by a female's wingbeat [10].

After successfully obtaining a blood-meal, female mosquitoes repress host-seeking behavior [11, 12], and utilize the nutrients in the blood-meal to develop a batch of eggs. A female who has reached this physiological state is known as "gravid". It is known that egg maturation and the beginning of egg-laying behavior occur between 48 and $96 \mathrm{~h}$ after a blood-meal [11]. Once the eggs have matured, a gravid female uses cues such as humidity and the presence and quality of liquid water to identify a suitable place to lay her eggs, a behavior also known as oviposition [13]. Following oviposition, a female mosquito recovers her attraction to hosts and seeks out new blood-meals to produce successive batches of eggs. This process, including host-seeking, egg maturation and oviposition, is known as the gonotrophic cycle [11]. Disease transmission by mosquitoes is driven by this cyclical nature of female biting behavior, as a mosquito must first bite an infected host before becoming competent to spread infection to subsequent hosts.

Genetic resources, such as those that have long existed for conventional model organisms, would greatly facilitate investigation into the mechanistic basis of behavior in mosquitoes. While there has been impressive progress in mosquito transgenesis and mutagenesis in the past 20 years $[3,8,12,14-22]$, the large size of the Ae aegypti genome $(\sim 1.3 \mathrm{~Gb})$ and large transposable element load ( 47 \%) present formidable challenges to genome assembly, physical mapping and annotation [23-26]. Despite the limitations imposed by incomplete annotation of new mosquito genomes, several studies have profiled gene expression in individual sensory organs in the mosquitoes Ae. aegypti [27-29], Culex quinquefasciatus [30], Anopheles gambiae [31-34], and Toxorhynchites amboinensis [34].

Our work builds on these efforts by incorporating biological replicates sequenced at greater depth and from many isolated tissues in parallel in both females at several behavioral states, and in males. This large dataset makes it possible to detect genes expressed at low levels or expressed in only a few neurons, and to identify differential gene expression with statistical confidence. Since the anatomical substrate of host-seeking, egglaying and other mosquito behaviors is likely to be distributed across several tissues, parallel transcriptional profiling of multiple tissues in a single study increases the likelihood of capturing the full repertoire of genes involved in these complex behaviors.

To generate a transcriptome of peripheral and central neural tissues (or "neurotranscriptome") in Ae aegypti, we performed Illumina mRNA-sequencing (RNA-seq) on RNA isolated from male and female tissues. Tissues sampled included the brain, antenna, maxillary palp, proboscis, abdominal tip, legs and female ovary. To understand the influence of blood-feeding state on gene expression, we performed RNA-seq on a subset of tissues in female mosquitoes at three time-points: prior to a blood-meal (non-blood-fed), at $48 \mathrm{~h}$ following a blood-meal (blood-fed), and at $96 \mathrm{~h}$ following a blood-meal (gravid).

This project, as part of the NIAID VectorBase Driving Biological Projects Initiative [35], set out to accomplish three major goals: 1) to improve the existing annotation of protein-coding genes in the Ae. aegypti genome and identify genes not found in the current genome assembly; 2) to catalogue gene expression at the resolution of single tissues in host-seeking female and male mosquitoes; and 3) to identify changes in gene expression that are correlated with blood-feeding state and its associated behavioral changes. This neurotranscriptome significantly enhances the Ae. aegypti genome annotation, and identifies a large number of genes whose expression is sexually dimorphic and/or variable across the female gonotrophic cycle. We anticipate that these data will drive studies of the genetic and neural circuit basis of host-seeking and egg-laying behavior in Ae. aegypti.

\section{Results}

We profiled Ae aegypti gene expression in tissues of males, and females at three points in their gonotrophic cycle (Fig. 1a). To confirm the distinct behavioral states associated with these time points in the mosquitoes used for our RNA-seq study, we measured responses to live hosts and carbon dioxide, and monitored female egglaying. A uniport olfactometer was used to measure female mosquito attraction to a human forearm, which was presented along with carbon dioxide $\left(\mathrm{CO}_{2}\right)$ to activate the mosquitoes [12]. Non-blood-fed females showed strong attraction to these host cues, while blood-fed females did not respond and gravid females showed only modest host attraction (Fig. 1b). Because the act of oviposition is correlated with a return to the state of active host-seeking [11], egg-laying was prevented prior to behavioral testing or tissue dissection by depriving females of access to water. We confirmed that oviposition behavior was normal in these animals by placing individual blood-fed and gravid female mosquitoes into oviposition vials and scoring the number of eggs laid over an $8 \mathrm{~h}$ period. As expected, blood-fed mosquitoes laid no eggs, but nearly all gravid mosquitoes did (Fig. 1c).

To determine whether the diminished responses to human hosts following blood feeding can be solely attributed to a reduction in sensitivity to $\mathrm{CO}_{2}$, we utilized a multi-insect three-dimensional flight-tracking system [8] to assess the response of a group of 20 female 


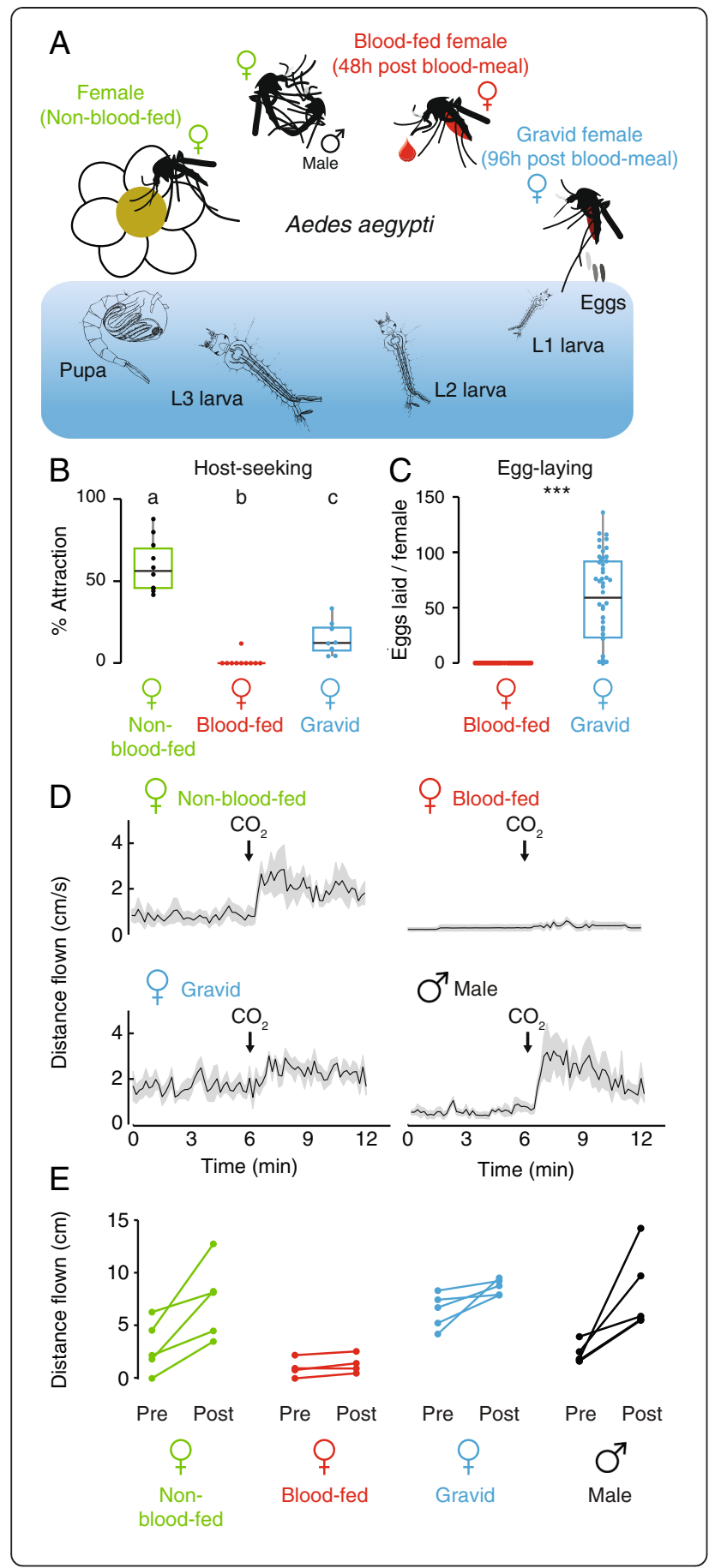

Fig. 1 Ae. aegypti life cycle and adult behaviors. a Schematic of the mosquito life cycle and the female gonotrophic cycle. $\mathbf{b}$ Female host-seeking behavior at three stages of the gonotrophic cycle as assayed in a uniport olfactometer [12] (ANOVA followed by Tukey's HSD, all differences $p<0.05, n=8-10$ per condition). c Eggs laid in 8 $\mathrm{h}$ after females were offered an oviposition substrate at 48 or $96 \mathrm{~h}$ after a blood-meal (Welch two-sample t-test, $p<0.001 ; n=50$ females). $\mathbf{d}$ Response to $\mathrm{CO}_{2}$ assayed in a three dimensional flight tracking arena [8]. Mean indicated with the black line and S.E.M. by the gray shading; $n=4-5$ per condition. e Summary of flight tracking data, with distance flown quantified in 6-min periods prior to and following a $40 \mathrm{~s}$ pulse of $\mathrm{CO}_{2}$. Post- $\mathrm{CO}_{2}$ activity of blood-fed females is reduced as compared to all other conditions (ANOVA followed by Tukey's HSD; $p<0.05$ ), and pre- $\mathrm{CO}_{2}$ activity of gravid females is increased as compared to all other conditions (ANOVA followed by Tukey's HSD; $p<0.05$ )

mosquitoes to a $40 \mathrm{~s}$ pulse of $\mathrm{CO}_{2}$ (Fig. 1d and e). As previously described, non-blood-fed female mosquitoes displayed a robust increase in flight activity in response to $\mathrm{CO}_{2}$ [8], while blood-fed females showed no increase in activity following administration of $\mathrm{CO}_{2}$. Gravid females displayed elevated pre- $\mathrm{CO}_{2}$ baseline flight activity. Male Ae. aegypti mosquitoes also showed a strong response to $\mathrm{CO}_{2}$ (Fig. 1d and e).

To study tissue-specific mRNA expression in isolated sensory and neural tissues of male and female $A e$. aegypti, we dissected individual tissues from pools of non-blood-fed, blood-fed and gravid female mosquitoes, as well as males. Tissue was flash-frozen and total RNA was extracted and used to generate Illumina RNA-seq libraries from polyA-selected mRNA. From female mosquitoes, we generated libraries from brain, antenna, proboscis, maxillary palp, foreleg, midleg, hindleg and ovary (Fig. 2a). We also dissected the rostrum, a tissue that includes both maxillary palp and proboscis, and the abdominal tip, defined in females as the three terminal abdominal segments, including genitalia and ovipositor (Fig. 2a). From male mosquitoes, we generated libraries from brain, antenna, rostrum, foreleg, midleg, hindleg and abdominal tip, defined in males as the three terminal abdominal segments, including genitalia (Fig. 2b). We generated at least three biological replicates for each tissue and subjected each to deep sequencing using an Illumina HiSeq instrument (with the exception of four libraries sequenced on the Illumina Genome Analyzer II) (Fig. 2c).

Correctly quantitating transcript expression from RNA-seq experiments depends on accurate gene models. Before analyzing gene expression across tissues, the two sexes and the female gonotrophic cycle, we utilized our sequencing data to update the annotation of proteincoding genes in the Ae. aegypti genome (Fig. 2d). The depth, replication and diversity of our sequencing allowed us to re-evaluate the existing annotation of protein-coding genes in the Ae. aegypti genome using 

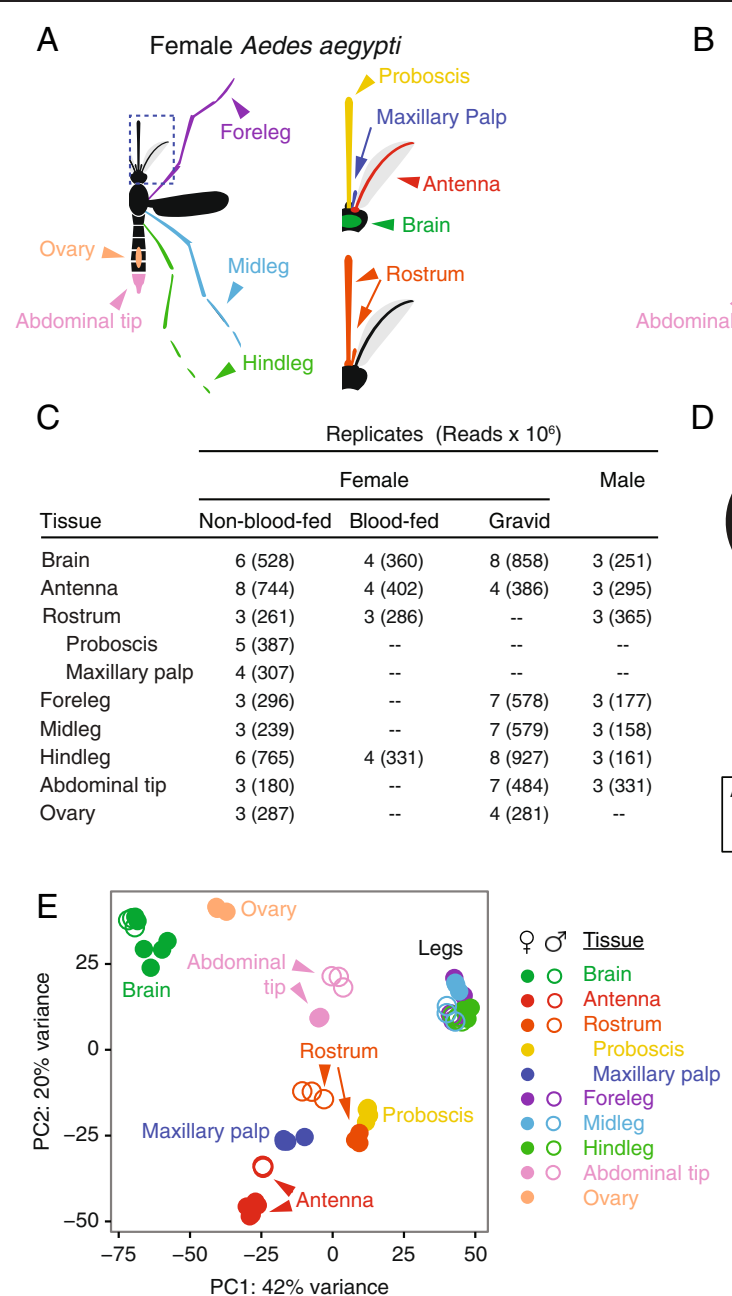

B Male Aedes aegypti

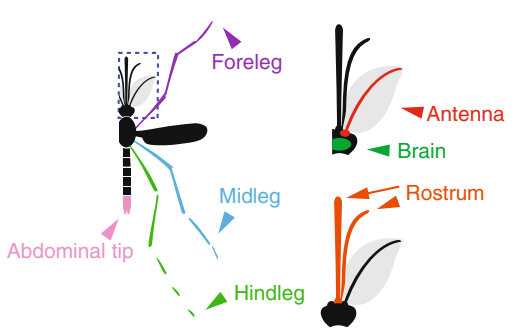

$\mathrm{D}$
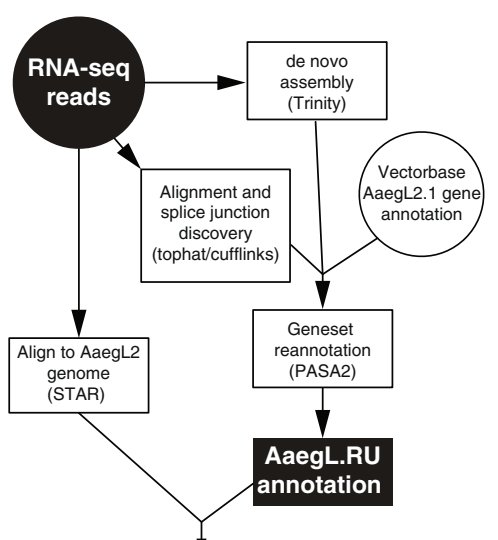

Gene counts

(featureCounts)

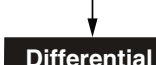

expression

(DESeq2)

Fig. 2 Neurotranscriptome tissues, sequencing and annotation methods. Cartoon of tissues dissected from adult female (a) and male (b) Ae. aegypti. The rostrum comprises maxillary palp, proboscis and connecting tissue. Number of biological replicates and sequencing reads across all conditions (c) and workflow chart (d) of analyses [36, 38-40, 86, 91] used to update the annotation of protein coding genes in the Ae. aegypti genome. e Principal component analysis of transcriptome-wide expression profiles of male and non-blood-fed female tissues

two complementary approaches: de novo transcriptome assembly using Trinity [36] and alignment of sequencing reads directly to the reference genome. By aligning contigs from the de novo assembly back to the genome, we were able to combine data generated from these two approaches and use PASA2 software [37] to update existing gene annotations (AaegL2.1; obtained from VectorBase [35]). Reads were also aligned to the genome using STAR [38], and those aligning to genes were counted using featureCounts [39], allowing us to estimate transcript abundance and calculate differential expression at the gene level using DESeq2 [40].

We first carried out a principal component analysis of male and non-blood-fed female libraries to examine the clustering of data by tissue and sex. Large batch effects from library construction methods or problems with tissue contamination during dissection [41] may be revealed by this process. Virtually all of the biological replicates of the same tissue clustered tightly in principal component space, and for brain and legs across the two sexes (Fig. 2e).

A comparison of the protein-coding transcriptomes from the community annotations AaegL2.1 and AaegL3.3 and our updated transcriptome, termed AaegL.RU, can be found in Fig. 3a-c. In addition to updating existing gene models, analysis identified 403 putative novel protein-coding genes that did not overlap with existing gene annotations and $>25,000$ new putative alternatively spliced isoforms as compared to the AaegL3.3 geneset (Fig. 3a and b). Furthermore, we identified 169 predicted protein-coding transcripts with orthology to other insect transcriptomes on unaligned contigs from our de novo assembly that likely derive from unsequenced portions of the Ae. aegypti genome 


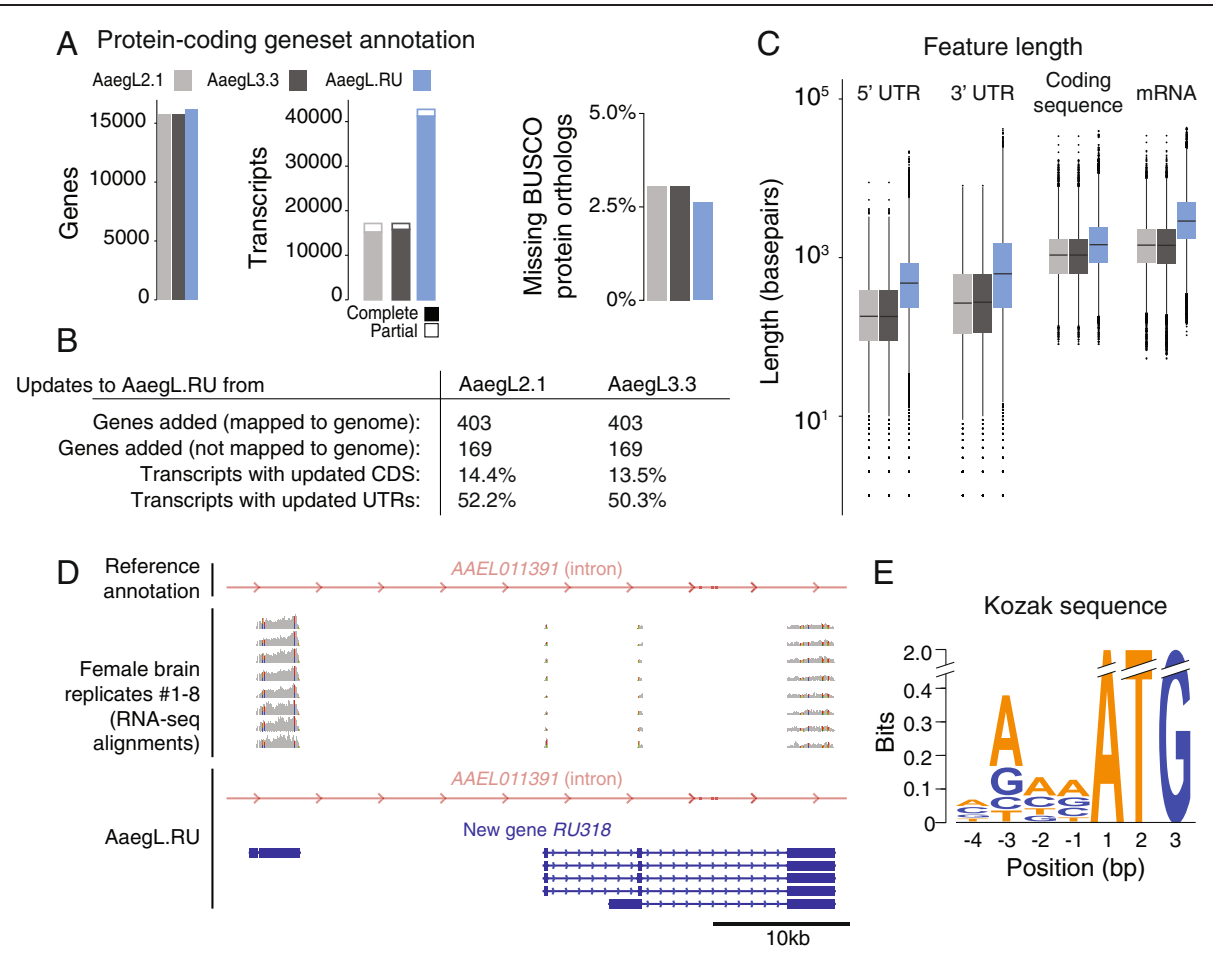

Fig. 3 Reannotation of the Ae. aegypti genome. a Annotation statistics comparing the base annotations (AaegL2.1 and AaegL3.3) with the updated AaegL.RU annotation. b Summary of added genes and novel predicted genomic loci and transcripts with updated coding sequences as determined by parseval. c Statistics of all predicted protein-coding transcripts, with data represented as box plots. Median indicated by black line, bounds of box represent first and third quartile, whiskers are 1.5 times the inter-quartile range and outliers denoted by dots. $\mathbf{d}$ RNA-seq evidence from 8 biological replicates supporting the existence of a PASA2-generated gene model for novel gene RU318 within an intron of existing gene model AAEL011391 on supercontig 1.575. e Sequence logo of the 4 bases upstream of the ATG start codon for all open reading frames from AaegL.RU, comprising the Kozak sequence

(Fig. 3b and Additional file 1). Our data also allowed for the extension of existing gene models, substantially increasing the length of UTRs and coding sequence for many existing genes as compared to the community reference annotations (Fig. $3 \mathrm{~b}$ and c). Together, we propose that there are potentially 572 protein-coding genes missing from the currently published annotation, although we note that our data must be integrated with and evaluated in the context of the large body of other genomic, transcriptomic and bioinformatic evidence available for Ae. aegypti [35]. The results of our reannotation, including novel transcripts, can be downloaded as Additional file 2 .

Using our updated geneset annotation, we next classified genes into families related to neuronal function by both incorporating previously published classifications as well as considering their relationship to genes in the well-annotated and -studied vinegar fly Drosophila melanogaster. For pre-existing genes, we identified their closest orthologue in D. melanogaster using precalculated orthology calls of OrthoDB [42]. To account for genes added in our geneset re-annotation, and thus not considered by the OrthoDB databases, we additionally performed blastx of the predicted coding sequence of all transcripts against the D. melanogaster proteome (Flybase release 6.06) and report the top BLAST hits with e-values below 0.01 (Additional file 3). Of note, 163 of our 572 proposed novel genes $(28.5 \%)$ have blastx hits that meet this criterion, as compared to $85.5 \%$ of all other annotated genes. Finally, we point out that due to the incomplete assembly of the Ae. aegypti genome $[23,43]$, ours and other approaches that rely on genomic coordinates to describe gene features will run the risk of duplication or error.

A single example of a novel protein-coding gene, $R U 318$, is depicted in Fig. 3d. It has high sequence conservation to the D. melanogaster TRP channel water witch (wtrw) [44]. Notably, the current Ae. aegypti geneset annotation lacks a predicted orthologue to water witch, while two other mosquito genomes (An. gambiae and $\mathrm{Cu}$. quinquefasciatus) contain orthologues. Based on this sequence similarity, we have included RU318 in our revised annotation of TRP channels in Ae. aegypti. Finally, to aid in transgenesis and other genome 
engineering approaches, we used all predicted coding sequences to generate a consensus Kozak sequence for $\mathrm{Ae}$. aegypti (Fig. 3e).

To describe transcript abundance across tissues, we mapped reads from our tissue-specific RNA-seq libraries to the AaegL3 genome and report transcript abundances in units of transcripts per million (TPM) [45]. Mapping statistics for each library can be found in Additional file 4 , and TPM values for each replicate library can be found in Additional file 5 and Additional file 6. We first described the expression of genes related to neuronal function in specific tissues of non-blood-fed female and male Ae. aegypti using expression values calculated against the AaegL.RU annotation.

Insects use three major neurotransmitters: acetylcholine is the primary excitatory neurotransmitter in the central nervous system, glutamate is used for neuromuscular transmission from motor neurons to muscles, and GABA is generally considered to be the primary inhibitory neurotransmitter in insect central synapses. Additionally, a number of biogenic amines including serotonin, dopamine, tyramine, octopamine and histamine function as neurotransmitters or neuromodulators.

We identified acetylcholine receptor subunits by orthology to D. melanogaster and An. gambiae [46]. Expression of most acetylcholine receptor subunits was highest in male and female brain (Fig. 4a). We profiled the expression of three types of glutamate receptors: Kainate, NMDA and AMPA (Fig. 4b). Overall, we found generally broader expression in peripheral tissues as well as brain, consistent with a role in neuromuscular transmission. Ionotropic GABA-A receptors and metabotropic GABA-B receptors were uniformly expressed broadly, with peaks in central brain (Fig. 4c). We found three histamine receptor orthologues: two most closely related to D. melanogaster HisCl1 and one to HisCl2 (Fig. 4d). All were expressed in brain at high levels, with the HisCl1 orthologue AAEL006047 also expressed broadly in peripheral tissues. Interestingly, we were unable to identify an orthologue of the glycine receptor Grd in Ae. aegypti. However, AAELO01568, annotated by Vectorbase as a potential glycine receptor subunit precursor, shows broad expression in peripheral tissues and more modest expression in brain (Fig. 4d).

Our annotation and a previous analysis [47] identified three genes likely to be involved in ammonia transport in $A$ e aegypti: two predicted orthologues of the $D$. melanogaster gene ammonium transporter (Amt), AAEL007373 and $A A E L 007377$, as well as a single orthologue of Rh50, AAEL008046. D. melanogaster Amt is required for the response of antennal coeloconic sensilla to ammonia [48]. Ammonia is known to be a host cue for Ae. aegypti [49] and An. gambiae [50, 51]. AAEL007377 was expressed at very low or undetectable levels (TPM $<1$ in all tissues) whereas AAELO07373 was expressed at high levels in female antenna and proboscis as well as male antenna (see Additional file 5). AAEL008046 was expressed at very high levels in male and female brain, as well as antenna (see Additional file 5). We propose that AAEL007373 and AAEL008046 may be involved in ammonia detection in host-seeking Ae. aegypti.

Biogenic amines represent an important class of neurotransmitters and neuromodulators in insects that have been implicated in processes as diverse as reward, aggression, oviposition choice and the control of context-specific social behavior [52-55]. Ae. albopictus mosquitoes fed constitutively with L-DOPA exhibit lower levels of host-seeking behavior [56]. Serotonin neurons innervate the antennal lobe of Ae. aegypti and An. gambiae [57], as well as the gut of Ae. aegypti [58] and serotonin has been shown to modulate feeding behavior in larval D. melanogaster [59]. Most serotonin receptors were expressed at appreciable levels in brain and in various peripheral tissues (Fig. 4e). Dopamine receptors had generally variable expression across tissues, including brain, legs and antennae (Fig. 4f). Octopamine/tyramine receptors had generally lower expression values than other receptors, but were detected in brain as well as peripheral tissues (Fig. $4 \mathrm{~g}$ ). The TyrR orthologue AAEL004396 was highly and selectively expressed in ovary. We generally observed little obvious sexual dimorphism in neurotransmitter receptor expression. This suggests that there is a gross conservation of neuronal cell types and signaling pathways, at the transcriptional level, across male and female tissues.

Neurotransmitter processing enzymes can serve as cell-type specific markers to reveal the major neurotransmitters produced in particular tissues. The orthologue of D. melanogaster glutamic acid decarboxylase 2, or Gad2, was expressed in brain and all peripheral sensory tissues, but not ovary. In contrast, Gad1 was largely restricted to brain, as well as male abdominal tip (Fig. 5a; Additional file 7). All dopamine processing enzymes were expressed in male and female brain, including the family of dopamine decarboxylase ( $D d c)$ genes, as well as tyrosine hydroxylase (TH), tyrosine decarboxylase $(T d c)$ and Tyramine $\beta$ hydroxylase (Tbh). We also observed strong peripheral expression of $T H$ and the two $D d c$ orthologues (Fig. 5a; Additional file 7). Processing enzymes associated with GABA, glutamate and acetylcholine were expressed in male and female brain, with many also expressed broadly across the majority of tissues sampled (Fig. 5a; Additional file 7).

Genes corresponding to Ae. aegypti neuropeptides and neuropeptide receptors were defined by orthology to canonical insect neuropeptides and receptors [12, 60-64]. Many neuropeptides were expressed primarily in brain of male and female mosquitoes, while a number had 


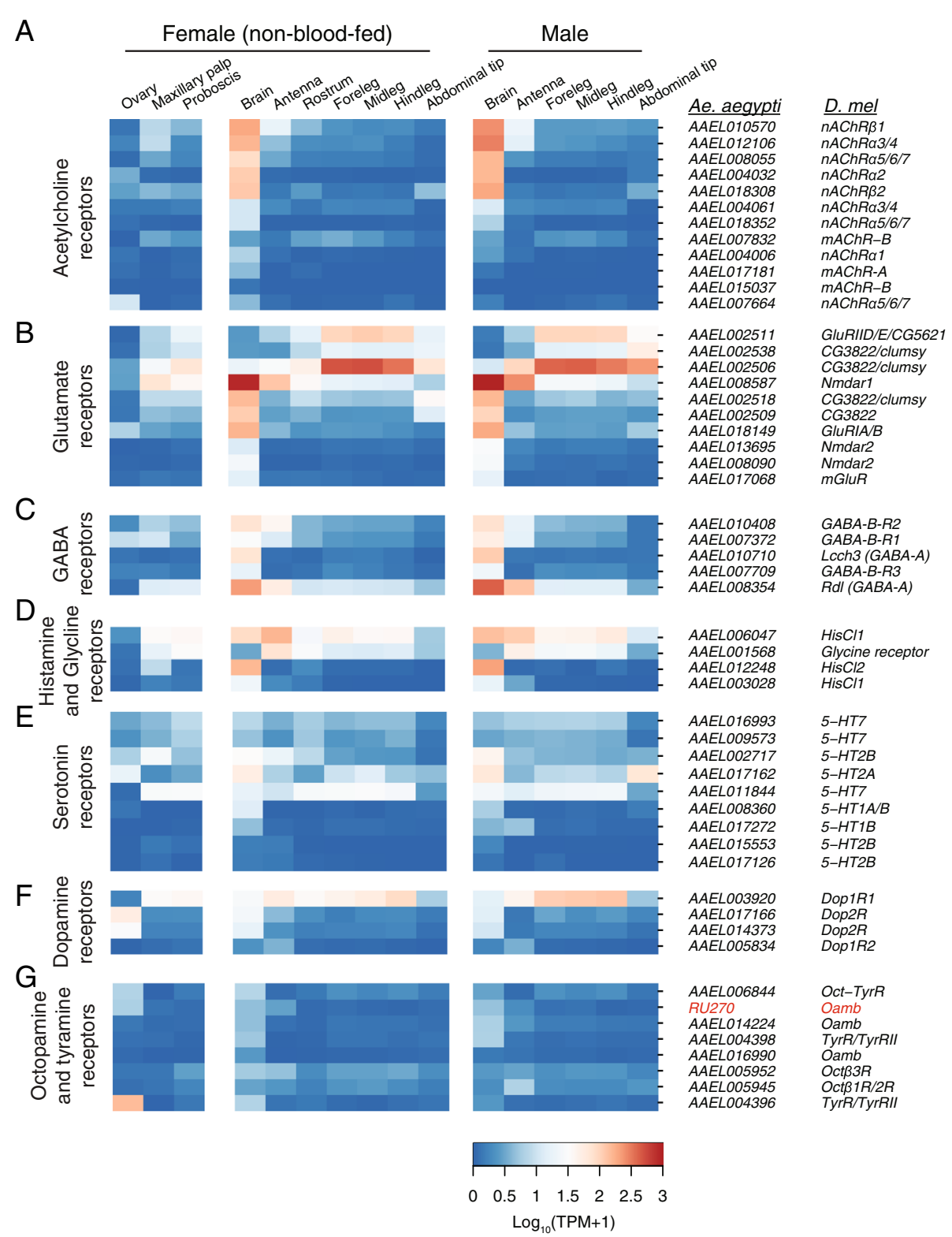

Fig. 4 Expression of neurotransmitter-related genes. Expression of receptors for the neurotransmitters acetylcholine (a), glutamate (b), GABA (c), histamine (d), serotonin (e), dopamine (f) and octopamine/tyramine $(\mathbf{g})$ in tissues from non-blood-fed female and male mosquitoes. Data are presented as the mean $\log _{10}(T P M+1)$ for all biological replicates from each tissue. AaegL.RU gene set names and the closest orthologue from D. melanogaster are indicated on the right

broader expression patterns that included but were not limited to brain (Fig. 5b; Additional file 7). This is consistent with reports of the direct detection of neuropeptides in specific regions of the brain, including the antennal lobe [65]. Other peptides, including several predicted orthologues of eclosion hormone $(E H)$, ecdysis-triggering hormone $(E T H)$ and bursicon, were not detected at appreciable levels. We speculate that these genes are expressed selectively in earlier developmental stages that were not sampled in the present study of adult tissues. Neuropeptide receptors had generally broad expression patterns (Fig. 5c; Additional file 7), indicating that neuropeptides may be centrally produced while exerting anatomically farreaching humoral effects.

Insects sense chemical substances such as tastants, odorants, pheromones, noxious chemicals and $\mathrm{CO}_{2}$ with an array of chemosensory receptors encoded by large gene families of odorant receptors (ORs), odorant binding proteins (OBPs), ionotropic receptors (IRs), gustatory receptors (GRs) and the pickpocket (PPK) and transient receptor potential (TRP) ion channels. Other 


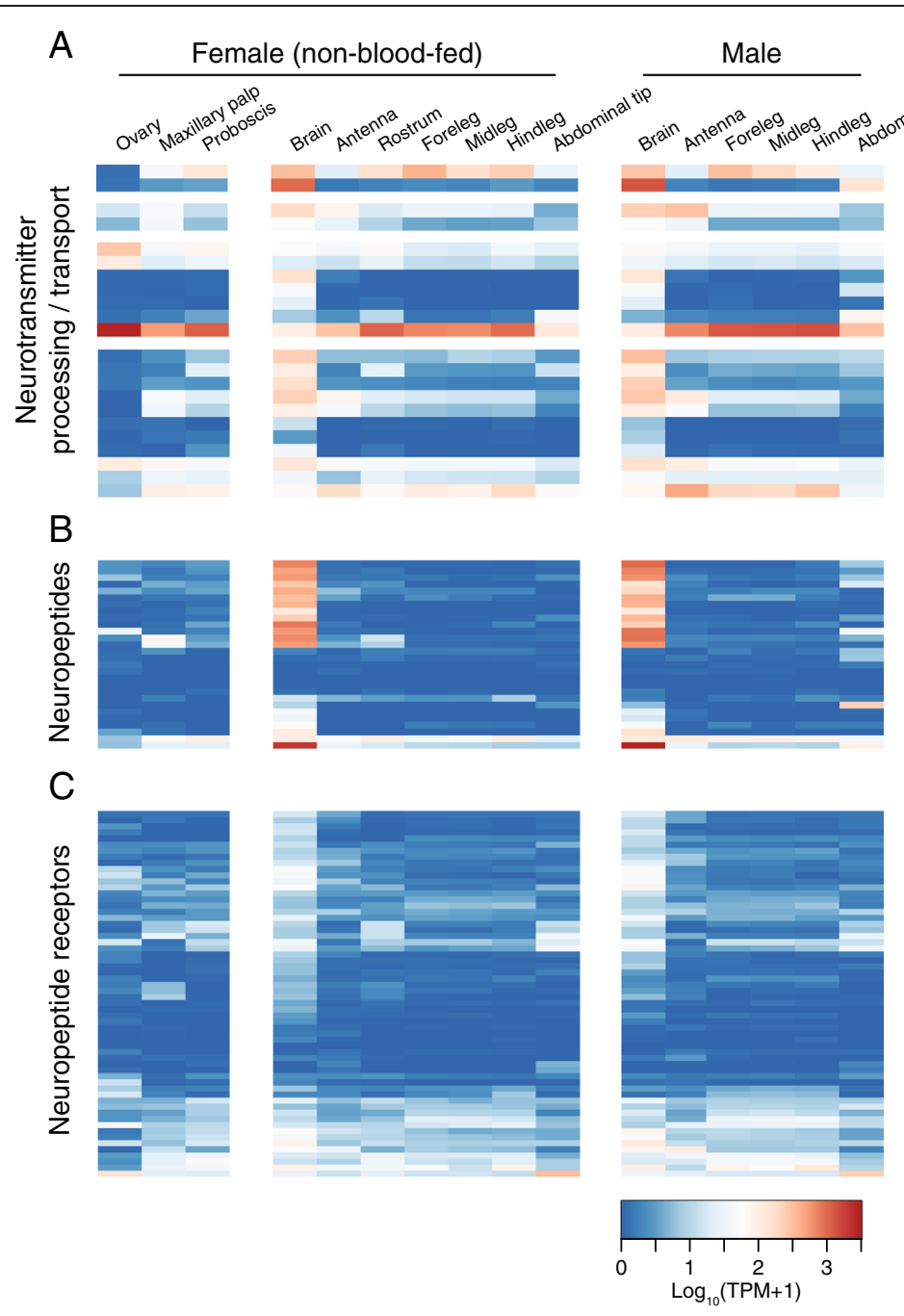

Fig. 5 Expression of biogenic amine-related genes. Expression of neurotransmitter transport and processing enzymes (a), neuropeptides (b) and neuropeptide receptors (c) in tissues from non-blood-fed female and male mosquitoes. Data are presented as the mean $\log _{10}$ (TPM +1 ) for all biological replicates from each tissue. AaegL.RU gene set names and the closest orthologue from D. melanogaster are provided in Additional file 7

groups have previously used RNA-seq to profile gene expression of a number of these genes in various tissues from several mosquito species [27, 28, 30-34]. Our work builds on these efforts by simultaneously profiling expression of all of these chemosensory genes in multiple individual tissues in Ae. aegypti in the same study (Figs. 6 and 7; Additional file 7).

ORs are an insect-specific family of divergent seven transmembrane domain chemoreceptors that sense volatile odors, including pheromones [66]. The majority of ORs are expressed in the antenna, with restricted subsets expressed in either proboscis or maxillary palp (Fig. 6a; Additional file 7), consistent with previous reports of OR-expressing sensory neurons in these tissues in An. gambiae [67] and Ae. aegypti [68]. In contrast to the ORs, OBPs are expressed widely in the tissues profiled here, and vary greatly in their transcript abundance (Fig. 6b; note expanded TPM scale relative to Fig. 6a; Additional file 7). Similar results were found in an analysis of OBP expression in An. gambiae mosquitoes [31] and ants [69].

Expression (TPM) values for ORs were broadly elevated in female as compared to male antenna. Antennae of male Ae. aegypti are specialized for audition and contain an exaggerated pedicel at their base when compared to female antenna [70]. We speculate that extra cell numbers associated with this enlarged pedicel would effectively dilute mRNA coming from other cells in male antenna, thus reducing the tissue-wide abundance of odorant receptors and other genes expressed in olfactory sensory neurons. To account for these putative differences, we plotted the expression of ORs in male and 

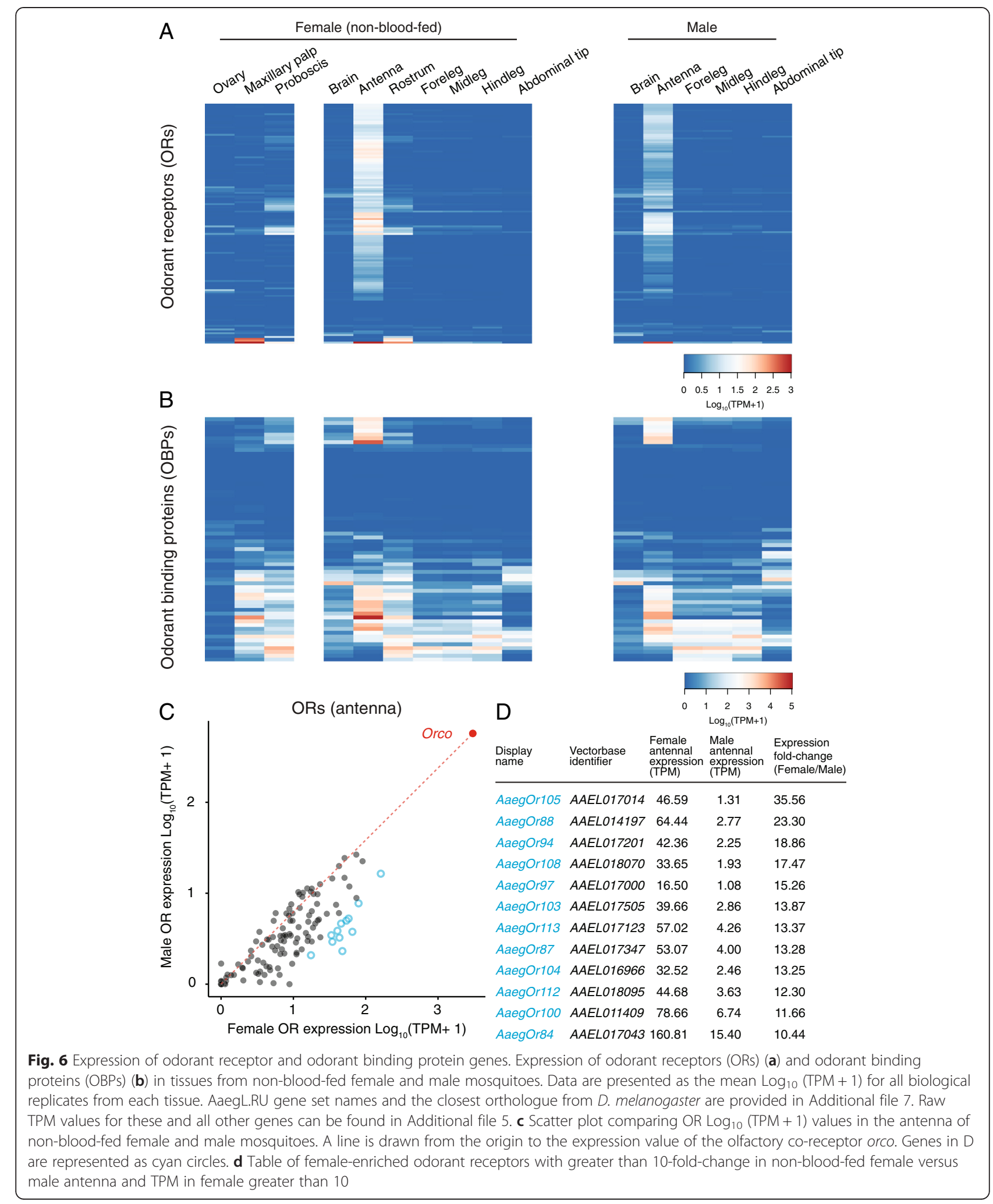

female antenna (Fig. 6c) normalized to the olfactory coreceptor orco to reflect the approximate number of olfactory sensory neurons. This normalization depends on the assumption that orco expression is not sexually dimorphic, and therefore a reasonable proxy for olfactory sensory neuron number across sexes. Even after 


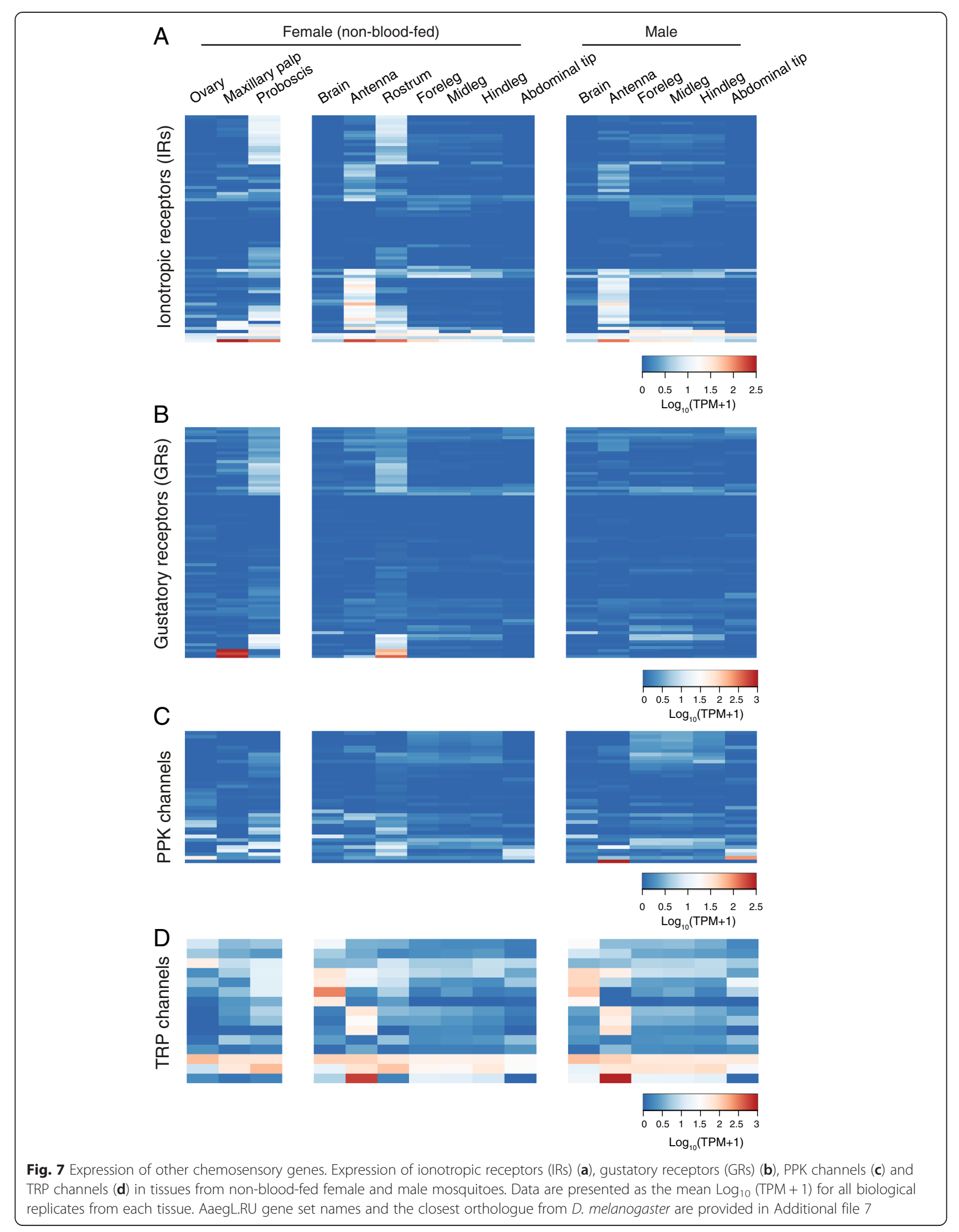


accounting for this normalization, we identified 12 OR genes with apparent enrichment in female antenna, with 10 - to 35 -fold increase in raw expression values as compared to male antennae (Fig. $6 \mathrm{c}$ and d). Because a number of untested assumptions were the basis of these conclusions, we note that these results would need to be validated independently, perhaps by RNA in situ hybridization, to gain cellular resolution of gene expression. Increases in mRNA expression could arise either by selective upregulation of gene expression in females, or through developmental changes that would lead to an increase in the number of neurons expressing these receptors in females. Because female mosquitoes show sexspecific chemosensory behavioral responses to odors associated with hosts and oviposition sites, it is not unreasonable to expect sex-specific differences in ORs tuned to these specific odors.

IRs are ligand-gated ion channels derived from variant ionotropic glutamate receptors that tend to be gated by ligands such as acids, aldehydes and amines [71]. The IR family of Ae. aegypti has been previously described [72], and we identified predicted gene models for 2 additional IRs (RU164 and RU199) using blastx against the D. melanogaster proteome (Additional file 2 and Additional file 3). We found three patterns of IR gene expression: those generally restricted to antenna; others selectively expressed in proboscis, rostrum and maxillary palp; and a small number of IRs expressed across many different tissues examined (Fig. 7a; Additional file 7). Similar results were previously reported in $D$. melanogaster, Apis mellifera, An. gambiae and Culex quinquefasciatus [30, 31, 72].

GRs are a family of transmembrane receptors distantly related to ORs [66] that mediate detection of pheromones, tastants, $\mathrm{CO}_{2}$ [73], and in the case of D. melanogaster Gr28b (Ae. aegypti Gr19), light and heat [74, 75]. The annotation of the GR family of Ae. aegypti was previously described [76]. GRs are predominantly expressed in the rostrum, maxillary palp and proboscis (Fig. 7b; Additional file 7), consistent with their primary and conserved role in taste perception [73]. Notable exceptions include AaegGr1, AaegGr2 and AaegGr3 (Fig. 7b, bottom 3 genes), which encode $\mathrm{CO}_{2}$ receptor genes that function in the maxillary palp [8].

Pickpocket (PPK) channels are a family of amiloridesensitive degenerin/epithelial sodium channels (DEG/ $\mathrm{ENaC}$ ) that are involved in the transduction of a number of sensory modalities, including mechanosensation, hygrosensation and pheromone sensing [77]. We first identified Ae. aegypti PPK channels by searching for orthologues to previously described PPKs in $D$. melanogaster and An. gambiae [77]. Gene expression profiles of the PPK channels reveal broad expression of most genes across several peripheral tissues (Fig. 7c; Additional file 7), including proboscis and legs, consistent with a role in various forms of contact chemosensation.

Transient receptor potential (TRP) channels have been implicated in diverse sensory modalities, including heat, light and chemosensation [78]. Ae. aegypti TRP channels were identified by conducting orthologue searches against the 13 identified D. melanogaster TRP channels [78]. We identified orthologues to all 13, and two additional genes predicted to be orthologues of $D$. melanogaster painless. Expression of TRP channels was generally broad (Fig. 7d), with several interesting tissue-specific expression patterns, most notably in brain and antenna.

In addition to profiling specific gene families, we examined transcripts with sexually dimorphic expression (Fig. 8, Additional file 8). We compared expression in 6 tissues between non-blood-fed females and males (Fig. 8a-f). Differences may arise from sexually dimorphic expression within individual cell-types or differences in tissue-specific cell-type composition between sexes. To identify a set of broadly dimorphic transcripts, we imposed a more conservative threshold of a foldchange greater than 8 in any tissues, and examined them for overlapping dimorphism across different tissues (Fig. $8 \mathrm{~g}$ and $\mathrm{h}$ ). Expression patterns of genes that were determined to be dimorphic in at least three tissues are indicated in gray in the Venn diagrams in Fig. $8 \mathrm{~g}$ and h, and displayed as heat maps in Fig. $8 \mathrm{i}$ and $\mathrm{j}$. We note the presence of two newly annotated RU genes in the malespecific transcript set, nix (RU468) [79] and RU185. myo-sex (RU529) [80] was identified as male-specific in two tissues. The discovery of these genes likely represents the ability of our RNA-seq data to capture transcripts produced from the Ae. aegypti male-specific locus on chromosome 1 that have eluded classical genome sequencing, and therefore annotation, due to large repetitive regions.

Finally, we examined changes in gene expression across the female gonotrophic cycle. After locating and biting a host, female mosquitoes become engorged on a blood-meal that can exceed their unfed body weight. Over the next few days, they must digest this blood and use its nutrients to mature a batch of eggs. During the first $48 \mathrm{~h}$ following a blood-meal, mosquitoes are less responsive to host cues and demonstrate very little locomotion overall (Fig. 1b, d and e) [11, 12]. Dramatic changes in gene expression in An. gambiae [32, 81], and olfactory function in An. gambiae and Ae. aegypti $[82,83]$ after a blood-meal have been documented. Despite these interesting observations, the mechanisms of host-seeking suppression following a blood-meal in Ae. aegypti are not well understood. To examine the 


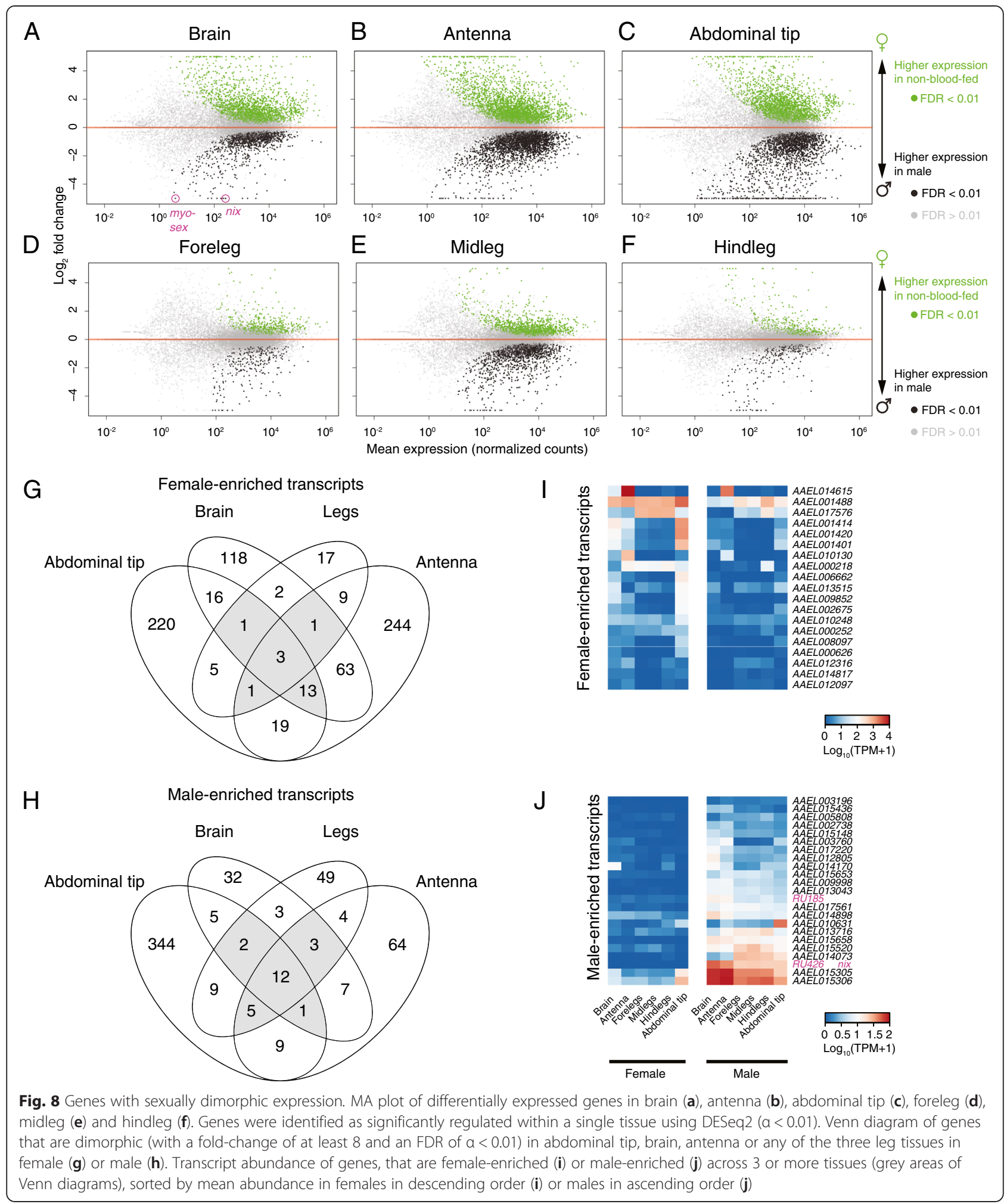

transcriptional changes associated with this behavioral shift, and begin to approach possible mechanisms, we asked whether there were significant changes in gene expression between non blood-fed, blood-fed and gravid mosquitoes in brain, antenna, hindleg (Fig. 9 and Additional file 9) and several other tissues (Additional file 9 and Additional file 10). Genes showing significant changes in expression are displayed in MA plots 


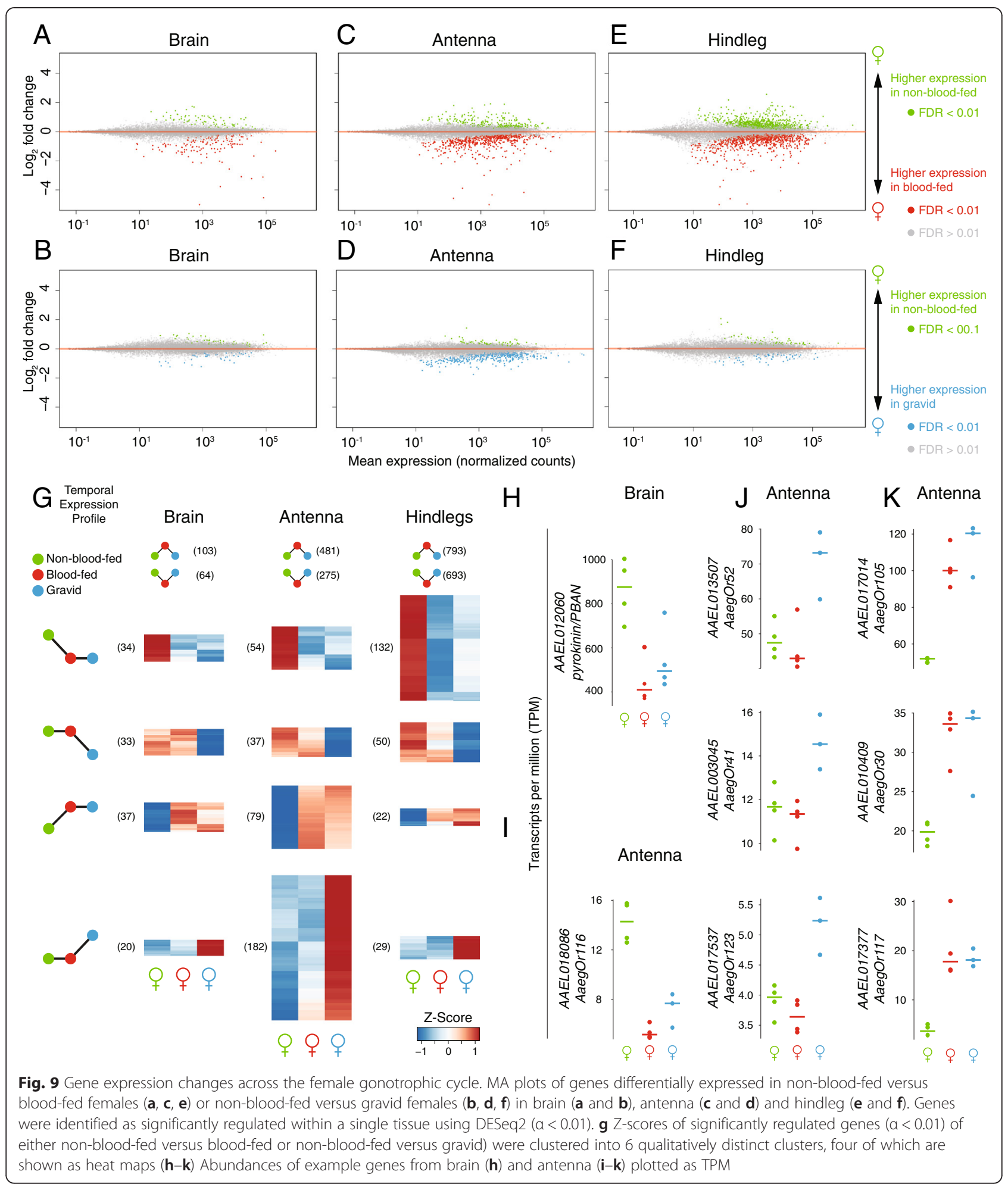

(Fig. 9a-f). We parsed these data to identify dynamics of gene expression across the gonotrophic cycle in these three tissues. Hundreds of genes showed selective up- and down-regulation in blood-fed relative to nonblood-fed females (Fig. 9g, top). Smaller numbers of genes showed peaks of expression in non-blood-fed animals, and suppression in blood-fed and gravid stages, mirroring the behavioral suppression observed in Fig. 1. We also identified sets of genes that showed lowest levels of expression at non-blood-fed or gravid stages, 
and those that had low levels of expression that later peaked in gravid animals. Selected examples of genes belonging to several of these classes of gene expression dynamics are shown in Fig. 9h-k, including the neuropeptide pyrokinin/PBAN (Fig. 9h) and 7 ORs (Fig. 9i-k).

\section{Discussion}

Here we present the "neurotranscriptome" of brain and peripheral nervous system tissues in female and male Ae. aegypti mosquitoes. We used both genome-guided mapping of RNA-seq reads as well as de novo transcriptome reconstruction to improve the annotation of existing protein-coding gene models as well as identify 572 putative novel protein-coding genes. By mapping tissuespecific RNA-seq libraries to transcripts generated by these updated gene models, we examined gene expression in 10 female and 6 male tissues from non-blood-fed animals, as well as a subset of tissues from blood-fed and gravid female mosquitoes, representing two important and distinct behavioral states following a human blood-meal.

Given the fragmented state of the Ae. aegypti genome and gene annotations, it was important to include a de novo assembly approach in our analysis. This allowed us to examine the expression pattern of genes derived from unassembled regions of the genome. For example, a myosin heavy chain gene, RU529, is identical in sequence to Ae. aegypti myo-sex [80], a gene linked to the sex-determining M-locus of Ae. aegypti in a region absent from the current genome assembly. A targeted search for genes in our dataset with similar expression patterns revealed additional genes with male-specific expression. Interestingly, we saw several novel unmapped genes from our de novo assembly in these searches and suggest that these may also derive from unassembled genomic loci similar to the M-locus. A recent study confirmed the presence of such a factor, nix (identified in our study as RU426), and demonstrated its critical role in sex-determination in Ae. aegypti [79].

The present study is a valuable dataset, presenting a comprehensive view of protein-coding gene expression in adult tissues, and yet it remains incomplete. We only sequenced polyadenylated RNA derived from 10 adult tissues. We have not explored the repertoire of small RNAs including microRNAs, non-coding RNAs or the regulation of alternative splicing. Our re-annotation approach relied on alignments of short reads and de novo transcripts back to the current draft of the genome, meaning that gene models residing on misassembled genomic contigs might be incorrectly represented. Indeed, a recent effort to generate a physical map for the Ae. aegypti genome found a misassembly rate of approximately $14 \%$, including 6 of the 10 largest supercontigs [23], making it likely that many gene models that rely on the present assembly remain incorrect. Ultimately, a comprehensive annotation of protein-coding genes and non-coding loci within the Ae. aegypti genome will require the incorporation of additional genomic sequencing and transcriptomic data derived from distinct developmental stages and tissues [84].

Whole tissue RNA-seq can identify genes differentially expressed across male and female tissues. However, further work will be required to resolve gene expression profiles in individual cells and cell-types. For the purposes of this study, sexually dimorphic transcripts were conservatively defined as those for which the foldchange observed was greater than 8 , though we note that there are many more transcripts with less extreme sex-biased expression. Anatomical differences will make it difficult to determine whether observed differences in transcript abundance represent differential regulation within shared cell-types or variation in the cell-type composition of male and female tissues. Interestingly, we describe relatively few examples of sexually dimorphic expression within the chemosensory gene families examined, suggesting that the striking behavioral differences seen between male and female Ae aegypti may be encoded in the neural circuits responsible for the processing of sensory stimuli as opposed to gene expression differences at the sensory periphery.

We do note the statistically significant up- and downregulation of a handful of olfactory receptors in antenna from gravid females. This is similar to an observed shift in OR expression in the antenna of An. gambiae following a blood-meal $[32,81]$, and suggests that a behavioral shift from host-seeking to oviposition site selection may involve the increased expression of particular ORs tuned to ligands associated with oviposition sites and a concomitant decrease in expression of ORs tuned to host odor. With few exceptions $[8,85]$, the ligand tuning of specific chemoreceptors has not been determined in Ae. aegypti. A systematic effort to de-orphanize $A e$. aegypti chemoreceptors will be required to address the functional relevance of these observed gene expression changes.

A major goal of this work was to identify gene expression changes correlated with blood-feeding state to gain insight into possible mechanisms by which a blood-meal might influence behavior. We describe many genes that change expression in tissues from blood-fed and gravid mosquitoes, including chemoreceptors, neuropeptides, neuropeptide receptors and neurotransmitter receptors and processing enzymes, all of which might play important roles in the regulation of behavior and physiology. However, genes from these classes comprise a small minority of all regulated genes, and thus, are unlikely to alone explain the marked shifts in behavior as female mosquitoes transition from host-seeking to oviposition. 
We envision this dataset as a resource to guide the selection of candidate genes involved in mosquito behavior as well as providing insight into the principles of gene expression regulation by blood-feeding. Transgenesis of mosquitoes [16] and precisely targeted mutagenesis with tools such as zinc-finger nucleases [3, 8, 12], TALENs $[18,19]$, homing endonucleases [17], and RNA-guided nucleases [20-22] now allow for the generation of stable mutant lines and other genetic reagents to test the function of candidate genes in mosquito behavior.

\section{Conclusions}

We present a broad view of gene expression in nonblood-fed male and female tissues, focusing particularly on gene families related to neuronal function and chemosensation. We demonstrate that the effects of bloodfeeding on gene expression are broad. This study represents the most comprehensive, tissue-specific survey of gene expression in adult Ae. aegypti to date and will be foundational in our understanding of the molecular genetic basis of behavior in this important disease vector.

\section{Methods}

\section{Mosquito rearing}

Mosquitoes used in this study were from the genome reference Liverpool strain (LVPIB12) obtained from BEI Resources/CDC/MR-4 (stock number MRA-735). Eggs were hatched in autoclaved water containing ground Tetramin tropical fish food (Tetra) and fed Tetramin food as necessary during larval and pupal development. For routine rearing, adult females were blood-fed on mice under a protocol approved by the Rockefeller University Institutional Animal Care and Use Committee (IACUC Protocol 14756). Male and female adult mosquitoes were reared together under a $14 \mathrm{~h}$ light:10 h dark cycle under conditions of $25-28{ }^{\circ} \mathrm{C}$ and $70-80 \%$ relative humidity. For female blood-fed libraries, mosquitoes were offered a human arm and allowed to feed to completion. Blood-feeding was verified by separating female mosquitoes with engorged abdomens 24-48 h following blood-feeding. At least $16 \mathrm{~h}$ prior to dissections, mosquitoes were separated under cold-anesthesia into groups of the appropriate size for a given library.

\section{Mosquito behavior}

Uniport experiments were carried out as described [12], with the exception of the stimulus, which was a $11 \mathrm{~cm}^{2}$ circle of exposed skin created by cutting a hole in an elbow-length latex glove. $\mathrm{CO}_{2}$ concentration in the airstream was measured at $5 \%$ with a Carbocap HandHeld $\mathrm{CO}_{2} \mathrm{~m}$ (model GM70, Vaisala Inc.). SciTrackS experiments were carried out as described [8]. Groups of 20 mosquitoes were placed into the flight arena, allowed to acclimate for $15 \mathrm{~min}$, and then presented with a $40 \mathrm{~s}$ pulse of $\mathrm{CO}_{2}$.

\section{Ethics, consent, and permissions}

All blood-feeding procedures and behavioral testing with human subjects were approved and monitored by The Rockefeller University Institutional Review Board (IRB; protocol LVO-0652). Subjects gave their written informed consent to participate in these experiments.

\section{Tissue dissection and RNA extraction}

Mosquitoes were cold-anesthetized and kept on ice until dissections were complete. Individual tissues were removed by forceps or scissors and immediately flashfrozen by placing into nuclease-free tubes in a dry-ice/ ethanol bath $\left(-76^{\circ} \mathrm{C}\right)$. The following number of mosquitoes was used for each female library: antenna, 100-220; maxillary palp, 126-816; proboscis, 275-797; rostrum, 110-142; brain, 9-18; foreleg, 125; midleg, 100-125; hindleg, 100-138; ovary, 9-25; abdominal tip, 50. For male libraries, the following number of mosquitoes was dissected for each tissue library: antenna, 75; rostrum, 40-50; brain, 25; foreleg, 100-125; midleg, 100-125; hindleg, 100-125; abdominal tip, 50. Dissected tissue was stored at $-80{ }^{\circ} \mathrm{C}$ until RNA extraction.

RNA extraction was performed using the Qiagen RNeasy Mini Kit (Qiagen). Tissue was disrupted with an electric tissue grinder loaded with a disposable RNAse free plastic pestle. For legs, abdominal tip and other cuticle-rich tissue, samples were further disrupted by passing tissue through a QIAshredder Mini spin column (Qiagen). RNA quantity and quality were evaluated using an Agilent BioAnalyzer 2100 and the RNA 6000 Nano Kit (Agilent Technologies).

\section{RNA-seq library preparation}

Unstranded libraries from polyA-selected RNA were prepared with TruSeq RNA Sample Preparation Kits (Illumina) or the mRNA sample-prep kit (Illumina), following the manufacturer's protocol. Between $200 \mathrm{ng}$ and $1 \mu \mathrm{g}$ of total RNA was used as input for each replicate library. For paired-end libraries, size-selection was performed prior to PCR by gel extraction or by a Pippin Prep instrument (Sage Biosciences) using $2 \%$ agarose cassettes containing ethidium bromide. Size selection resulted in libraries with mean insert sizes (excluding sequencing adapters) of 250-450 base pairs (bp). Library quantity and quality were evaluated using an Agilent BioAnalyzer 2100 and the High Sensitivity DNA Kit.

\section{Sequencing}

All sequencing was performed at The Rockefeller University Genomics Resource Center on HiSeq 2000 or Genome Analyzer IIx sequencers (Illumina). All paired 
end reads were $2 \times 101 \mathrm{bp}$ and all single-end reads were $1 \times 101$ bp with the exception of four 72 bp libraries. Data were de-multiplexed and delivered as fastq files for each library. These sequencing reads are available at the NCBI Sequence Read Archive (SRA) and are associated with BioProject PRJNA236239.

\section{Transcriptome generation: reference-based mapping}

All reads from all libraries were aligned to the AaegL2 reference genome obtained from VectorBase [35] using Cufflinks2, Tophat2 and Bowtie2 software packages [86]. Reads were aligned without respect to existing annotations with the following settings: minimum intron length of $40 \mathrm{bp}$, maximum intron length of $500 \mathrm{Mb}$. Cufflinks was run on reads from individual conditions and tissues to identify all putative splice junctions, and then combined using cuffcompare to identify a consensus set of putative splice junctions identified in our sequencing reads.

\section{Transcriptome generation: de novo assembly}

We performed de novo assembly as a second approach to reconstruct transcripts from our data. All reads from all libraries were assembled into a genome-free de novo assembly using the Trinity software package (version 2013-02-25) [36, 87]. To account for the depth of sequencing, we first performed read normalization to down-sample the number of reads used in the assembly using the included normalization tool in the Trinity software package with a max_coverage setting of 25. Male paired-end, female paired-end and single-end reads were normalized separately and then combined, resulting in a 49-fold reduction in overall input data. Trinity was run using default settings, with a minimum k-mer coverage of 1 , resulting in an assembly with 420,978 contigs.

\section{Geneset annotation: PASA}

The spliced alignments of individual sequencing reads and the alignment of contigs from the de novo assembly were used as input to PASA2 [37] as a means of updating the reference gene annotation using the software's alignment assembly and annotation comparison workflow. Briefly, de novo contigs were aligned to the genome (AaegL2; VectorBase [35]) using the short read aligners BLAT and GMAP. These alignments were combined with the combined cufflinks output from genome-guided mapping to create assemblies of spliced alignments. These assemblies were compared to reference annotations (AaegL2.1; VectorBase [35]) and used to extend, update or merge reference annotations. Additionally, this analysis identified 403 putative protein-coding genes not covered by the current annotation (see "Geneset annotation: naming of genes and geneset comparisons below). Default PASA2 parameters were used with the exception of the number of allowed exons in 5' or 3' UTRs (--MAX_UTR_EXONS =3). Due to a technical oversight, 5 genes were added manually after the PASA2 run, using previously published coordinates: AaegGr27, AaegOr54, AaegIr41d.2, AaegIr75k.4 and AaegIr7h.2.

\section{Identifying novel unmapped genes}

To identify novel transcripts that do not map to the current genome assembly, we filtered our de novo assembly as follows. First, we excluded all contigs that mapped to the genome or to cDNA from an existing transcript using GMAP. Next, we required that each contig encoded a complete open reading frame (ORF) of at least 30 amino acids in length, as predicted by transdecoder (http://transdecoder.github.io). We then screened for likely bacterial and fungal contamination by performing blastx with default settings of the remaining contigs against the $\mathrm{nr}$ database (NCBI), and excluded anything for which the top hit was fungal, bacterial or mammalian. Finally, we performed blastx of each remaining contig against a database of insect transcriptomes (Anopheles gambiae [AgamP3], Apis mellifera [Amel_4.0], Culex pipiens (now Culex quinquefasciatus) [CpipJ1], Drosophila pseudoobscura [r3.1], Heliconius melpomene [v1.1], Ixodes scapularis [IscaW1.2], Nasonia vitripennis [Nvit_1.0], Rhodnius proxlixus [RproC1], Bombyx mori [SilkDB v1.0], Drosophila melanogaster [r5.50] and Triboleum castaneum [v2.0; without mitochondria], requiring that there was a match with an evalue of less than 0.01. 232 contigs that passed these conservative filters were considered to be highconfidence novel genes derived from portions of the genome that have not been sequenced or had assembly problems. These 232 contigs were collapsed using CDHIT $[88,89]$ resulting in 169 novel transcripts that were included in downstream analysis.

\section{Geneset annotation: naming of genes and geneset comparisons}

To name each gene in our updated geneset, we first compared them to existing annotations in AaegL3.3 using cuffcompare [86] and carried over accession numbers for those loci that were highly similar to existing annotations. Genes that did not match existing loci in these cuffcompare analyses are numbered sequentially as RU1-RU572 (Additional file 2). For chemosensory gene families with previously published manual annotation, names were assigned to be consistent with these previous annotations (Additional file 3). For genes with a VectorBase accession number, orthology to Drosophila melanogaster was retrieved from OrthoDB (ODB8, dipteran dataset) [42]. We further used NCBI blastx to compare CDS for all transcripts against the D. melanogaster proteome; hits with an e-value of less than 0.01 
(truncated to include the top 10) are listed in Additional file 3. To determine the proportion of transcripts that were updated as compared to the VectorBase annotations (Fig. 3c; AaegL2.1 and AaegL3.3), we used the parseval tool in the AEGeAn package to compare gff3 annotation files [90].

We note that due to the evolving nature of annotations in Ae. aegypti and the fact that our orthology relies only on a single species (D. melanogaster), the gene families here should not be considered complete or exhaustive.

\section{Library QC}

A file detailing library alignment and quantitation statistics is available as Additional file 4. While all sequencing reads were used for genome reannotation, aberrant clustering of transcriptome-wide expression patterns from two non-blood-fed female brain libraries resulted in their exclusion pool and the DESeq2 model was re-run. Additionally, signs of contamination of male rostrum libraries resulted in their removal from expression analysis (note that these are retained in the PCA plot in Fig. 2e).

\section{Expression data and differential expression analysis}

All reads from individual libraries were mapped to the AaegL3 genome using STAR version 2.4.1c [38], and reads mapping to each gene in the AaegL.RU or AaegL3.3 geneset annotation were counted at the gene level using featureCounts v1.4.6-p3 [39] (Additional file 3). For abundance visualization, raw counts were converted to TPM [45] in R. Raw counts were used for differential expression analysis in $\mathrm{R}$ using DESeq2 v1.8.2 [40], and the PCA analysis in Fig. 2e was performed with DESeq2 using counts subjected to Variance Stabilizing Transformation (VST).

Sexually dimorphic genes were identified with a DESeq2 model incorporating all non-blood-fed female and male libraries from a single tissue and visualized as MA plots generated with significance indicated at an FDR of $\alpha<0.01$ (Fig. 8a-f, Additional file 8). Venn diagrams were generated using the $\mathrm{R}$ library VennDiagram (Fig. 8g and h). Transcript abundance of genes identified as dimorphic in at least three tissue groups were visualized as heat maps sorted by the sum of the TPM in the dominant sex (Fig. 8i and j).

Genes regulated by blood-feeding state were identified in DESeq2 with single-tissue models incorporating single-end libraries from female tissues (with the exception of ovary, where all libraries were paired-end). In tissues with three time-points (brain, antenna and hindleg), Z-scores of expression for genes with an FDR of $\alpha$ $<0.01$ (in either comparison) were generated using the 'scale' function of $\mathrm{R}$, and clustered using the hclust
( $\operatorname{method}=$ 'complete') and dist ('method $=$ euclidean') functions in R. (Fig. 9).

\section{Availability of data and materials}

All raw reads are deposited in the NCBI SRA under BioProject number PRJNA236239. Gene set annotations, expression data and sequences of new genes generated are available as Additional Files with this manuscript.

\section{Additional files}

\section{Additional file 1: Is a FASTA file with the raw sequences for} unmapped genes. (FA $277 \mathrm{~kb}$ )

Additional file 2: Is a GFF3 file with the complete annotation of all genes in the AaegL.RU geneset. (GFF3 90.8 mb)

Additional file 3: Is an annotation table for all genes in AaegL.RU, including OrthoDB orthologue groups shared with $D$. melanogaster. (XLSX $841 \mathrm{~kb}$ )

Additional file 4: Describes the mapping and read count statistics for each individual RNA-seq library. (XLSX $24 \mathrm{~kb}$ )

Additional file 5: Is a table of transcript abundance (in units of TPM) for all non-blood-fed and male tissues, AaegL.RU annotation. (XLSX 20937 kb)

Additional file 6: Is a table of transcript abundance (in units of TPM) for all non-blood-fed and male tissues, AaegL3.3 annotation. (XLSX 21243 kb)

Additional file 7: Shows an expansion of expression heat-maps from Figs. 5 to 7 showing gene names for individual rows. (PDF $463 \mathrm{~kb}$ )

Additional file 8: Is a spreadsheet describing the DESeq2 differential expression statistics of sexually dimorphic transcripts for all applicable tissues. (XLSX $1366 \mathrm{~kb}$ )

Additional file 9: Is a spreadsheet containing the DESeq2 differential expression statistics of transcripts regulated by blood-feeding state for all applicable tissues. (XLSX 1852 kb)

Additional file 10: Shows gene expression changes across the female gonotrophic cycle. MA plots of genes differentially expressed in non-blood-fed versus blood-fed female rostrum (a) or non-blood-fed versus gravid female forelegs (b), midlegs (c), ovary (d) and abdominal tip (e). Genes were identified as significantly regulated by a single-tissue comparison using DESeq2 ( $a<0.01$ ). (PDF 3876 kb)

\section{Abbreviations}

5-HT: 5-hydroxytryptamine (serotonin); bp: base pair; CDS: coding sequence; $\mathrm{CO}_{2}$ : carbon dioxide; $\mathrm{CV}$ : coefficient of variation; DA: dopamine;

Ddc: dopamine decarboxylase; DEG/ENaC: degenerin/epithelial sodium channels; DNA: deoxyribonucleic acid; EH: eclosion hormone; EST: expressed sequence tag; ETH: ecdysis-triggering hormone; GABA: $\gamma$-aminobutryic acid; IACUC: Institutional Animal Care and Use Committee; IR: ionotropic receptor; IRB: Institutional Review Board; kb: kilobase; m: meter; MA plot: plot using an $M$ (log ratios) and $A$ (mean average) scale; Mb: megabase; $\mathrm{mL}$ : milliliter; NPY: neuropeptide Y; OBP: odorant binding protein; OR: odorant receptor; ORF: open reading frame; PCA: principal component analysis;

PCR: polymerase chain reaction; PPK: pickpocket; RNA: ribonucleic acid; RNA-seq: mRNA-sequencing; sec: seconds; Tbh: Tyramine $\beta$ hydroxylase; Tdc: tyrosine decarboxylase; Th: tyrosine hydroxylase; TPM: transcripts per million; TRP: transient receptor potential; $\mu \mathrm{L}$ : microliter; UTR: untranslated region; VST: variance stabilizing transformation; wtrw: water witch.

\section{Competing interests}

The authors declare that they have no competing interests. 


\section{Authors' contributions}

BJM, CSM and MJD dissected tissues and made libraries and together with LBV conceived and planned the study; BJM carried out the experiments in Fig. 1; BJM, CSM and OD carried out bioinformatic analysis. BJM and LBV wrote the paper and produced the figures, with input from the other authors. All authors have read and approved the manuscript.

\section{Acknowledgements}

We thank Nicolas Robine, and members of the Vosshall Lab for discussions and comments on the manuscript; Felix Baier and Chloe Goldman for expert technical assistance; Katie Kistler for assisting with the behavioral experiments in Fig. $1 \mathrm{~b}$ and c; Deborah Beck and Gloria Gordon for mosquito rearing; Daniel Lawson for discussion and sharing annotations in progress at VectorBase; Connie Zhao and James Hughes for facilitation of DNA sequencing at the Rockefeller University Genomics Resource Center, and Scott Dewell for bioinformatic support. This work was funded in part by a grant to R. Axel and L.B.V. from the Foundation for the National Institutes of Health through the Grand Challenges in Global Health Initiative and the following National Institutes of Health grants: K99 award from NIDCD to CSM (DC012069), an NIAID VectorBase DBP subcontract to LBV

(HHSN272200900039C), and a CTSA award from NCATS (5UL1TR000043). BJM was supported by Henry and Marie-Josée Kravis and Jane Coffin Childs Postdoctoral Fellowships. LBV is an investigator of the Howard Hughes Medical Institute.

\section{Author details}

'Laboratory of Neurogenetics and Behavior, The Rockefeller University, New York, NY 10065, USA. ${ }^{2}$ Howard Hughes Medical Institute, New York, NY 10065, USA. ${ }^{3}$ Present Address: Department of Ecology and Evolutionary Biology, Princeton Neuroscience Institute, Princeton University, Princeton, NJ 08544, USA. ${ }^{4}$ Present Address: Department of Biological Sciences, Biomolecular Sciences Institute, Florida International University, Miami, FL 33199, USA. ${ }^{5}$ Present address: Stanford University, Stanford, CA 94305, USA.

Received: 3 September 2015 Accepted: 24 November 2015

Published online: 06 January 2016

\section{References}

1. Bhatt S, Gething PW, Brady OJ, Messina JP, Farlow AW, Moyes CL, et al. The global distribution and burden of dengue. Nature. 2013;496:504-7.

2. Christophers SR. Aedes aegypti (L.) the yellow fever mosquito: its life history, bionomics and structure. Cambridge: Cambridge University Press; 2009.

3. DeGennaro M, McBride CS, Seeholzer L, Nakagawa T, Dennis EJ, Goldman C, et al. orco mutant mosquitoes lose strong preference for humans and are not repelled by volatile DEET. Nature. 2013;498:487-91.

4. Gibson G, Torr SJ. Visual and olfactory responses of haematophagous Diptera to host stimuli. Med Vet Entomol. 1999:13:2-23.

5. Gillies MT. The role of carbon dioxide in host-finding by mosquitoes (Diptera: Culicidae): a review. Bull Entomol Res. 1980;70:525-32.

6. Peterson DG, Brown AWA. Studies of the responses of the female Aëdes mosquito. Part III. The response of Aëdes aegypti (L.) to a warm body and its radiation. Bull Entomol Res. 1951;42:535-41.

7. van Breugel F, Riffell J, Fairhall A, Dickinson MH. Mosquitoes use vision to associate odor plumes with thermal targets. Curr Biol. 2015;25(16):2123-9.

8. McMeniman CJ, Corfas RA, Matthews BJ, Ritchie SA, Vosshall LB. Multimodal integration of carbon dioxide and other sensory cues drives mosquito attraction to humans. Cell. 2014;156:1060-71.

9. Hartberg WK. Observations on the mating behaviour of Aedes aegypti in nature. Bull World Health Organ. 1971;45:847-50.

10. Cator $L$, Arthur BJ, Harrington LC, Hoy RR. Harmonic convergence in the love songs of the dengue vector mosquito. Science. 2009;323:1077-9.

11. Klowden MJ. Initiation and termination of host-seeking inhibition in Aedes aegypti during oöcyte maturation. J Insect Physiol. 1981;27:799-803.

12. Liesch J, Bellani LL, Vosshall LB. Functional and genetic characterization of neuropeptide Y-like receptors in Aedes aegypti. PLoS Negl Trop Dis. 2013;7:e2486

13. Hudson BNA. The behaviour of the female mosquito in selecting water for oviposition. J Exp Biol. 1956:33:478-92.

14. Sarkar A, Yardley K, Atkinson PW, James AA, O'Brochta DA. Transposition of the Hermes element in embryos of the vector mosquito, Aedes aegypti. Insect Biochem Mol Biol. 1997;27:359-63.
15. Jasinskiene N, Coates CJ, Benedict MQ, Cornel AJ, Rafferty CS, James AA, et al. Stable transformation of the yellow fever mosquito, Aedes aegypti, with the Hermes element from the housefly. Proc Natl Acad Sci U S A. 1998:95:3743-7.

16. Lobo NF, Clayton JR, Fraser MJ, Kafatos FC, Collins FH. High efficiency germline transformation of mosquitoes. Nat Protoc. 2006;1:1312-7.

17. Aryan A, Anderson MA, Myles KM, Adelman ZN. Germline excision of transgenes in Aedes aegypti by homing endonucleases. Sci Rep. 2013;3:1603.

18. Aryan A, Anderson MA, Myles KM, Adelman ZN. TALEN-based gene disruption in the dengue vector Aedes aegypti. PLoS One. 2013;8:e60082.

19. Aryan A, Myles KM, Adelman ZN. Targeted genome editing in Aedes aegypti using TALENs. Methods. 2014;69(1):38-45.

20. Kistler KE, Vosshall LB, Matthews BJ. Genome engineering with CRISPR-Cas9 in the mosquito Aedes aegypti. Cell Rep. 2015;11:51-60.

21. Basu S, Aryan A, Overcash JM, Samuel GH, Anderson MA, Dahlem TJ, et al. Silencing of end-joining repair for efficient site-specific gene insertion after TALEN/CRISPR mutagenesis in Aedes aegypti. Proc Natl Acad Sci U S A. 2015;112:4038-43.

22. Dong S, Lin J, Held NL, Clem RJ, Passarelli AL, Franz AW. Heritable CRISPR/ Cas9-mediated genome editing in the yellow fever mosquito, Aedes aegypti. PLoS One. 2015:10:e0122353.

23. Juneja P, Osei-Poku J, Ho YS, Ariani CV, Palmer WJ, Pain A, et al. Assembly of the genome of the disease vector Aedes aegypti onto a genetic linkage map allows mapping of genes affecting disease transmission. PLoS Negl Trop Dis. 2014;8:e2652.

24. Nene V, Wortman JR, Lawson D, Haas B, Kodira C, Tu ZJ, et al. Genome sequence of Aedes aegypti, a major arbovirus vector. Science. 2007;316: 1718-23.

25. Severson DW, Behura SK. Mosquito genomics: progress and challenges. Annu Rev Entomol. 2012;57:143-66.

26. Timoshevskiy VA, Severson DW, Debruyn BS, Black WC, Sharakhov IV Sharakhova MV. An integrated linkage, chromosome, and genome map for the yellow fever mosquito Aedes aegypti. PLoS Negl Trop Dis. 2013;e2052.

27. Bohbot JD, Sparks JT, Dickens JC. The maxillary palp of Aedes aegypti, a model of multisensory integration. Insect Biochem Mol Biol. 2014;48:29-39.

28. Sparks JT, Bohbot JD, Dickens JC. The genetics of chemoreception in the labella and tarsi of Aedes aegypti. Insect Biochem Mol Biol. 2014;48:8-16.

29. Sparks JT, Vinyard BT, Dickens JC. Gustatory receptor expression in the labella and tarsi of Aedes aegypti. Insect Biochem Mol Biol. 2013;43:1161-71.

30. Leal WS, Choo YM, Xu P, da Silva CS, Ueira-Vieira C. Differential expression of olfactory genes in the southern house mosquito and insights into unique odorant receptor gene isoforms. Proc Natl Acad Sci U S A. 2013;1 10:18704-9.

31. Pitts RJ, Rinker DC, Jones PL, Rokas A, Zwiebel LJ. Transcriptome profiling of chemosensory appendages in the malaria vector Anopheles gambiae reveals tissue- and sex-specific signatures of odor coding. BMC Genomics. 2011;12:271.

32. Rinker DC, Pitts RJ, Zhou X, Suh E, Rokas A, Zwiebel LJ. Blood meal-induced changes to antennal transcriptome profiles reveal shifts in odor sensitivities in Anopheles gambiae. Proc Natl Acad Sci U S A. 2013;110:8260-5.

33. Rinker DC, Zhou X, Pitts RJ, Consortium AGC, Rokas A, Zwiebel LJ. Antennal transcriptome profiles of anopheline mosquitoes reveal human host olfactory specialization in Anopheles gambiae. BMC Genomics. 2013;14:749.

34. Zhou X, Rinker DC, Pitts RJ, Rokas A, Zwiebel LJ. Divergent and conserved elements comprise the chemoreceptive repertoire of the nonblood-feeding mosquito Toxorhynchites amboinensis. Genome Bio Evol. 2014;6:2883-96.

35. Giraldo-Calderon Gl, Emrich SJ, MacCallum RM, Maslen G, Dialynas E, Topalis $P$, et al. VectorBase: an updated bioinformatics resource for invertebrate vectors and other organisms related with human diseases. Nucleic Acids Res. 2015:43:D707-13.

36. Grabherr MG, Haas BJ, Yassour M, Levin JZ, Thompson DA, Amit I, et al. Full-length transcriptome assembly from RNA-Seq data without a reference genome. Nat Biotechnol. 2011;29:644-52.

37. Haas BJ, Salzberg SL, Zhu W, Pertea M, Allen JE, Orvis J, et al. Automated eukaryotic gene structure annotation using EVidenceModeler and the Program to Assemble Spliced Alignments. Genome Biol. 2008;9:R7.

38. Dobin A, Davis CA, Schlesinger F, Drenkow J, Zaleski C, Jha S, et al. STAR: ultrafast universal RNA-seq aligner. Bioinformatics. 2013;29:15-21.

39. Liao Y, Smyth GK, Shi W. featureCounts: an efficient general purpose program for assigning sequence reads to genomic features. Bioinformatics. 2014;30:923-30.

40. Love MI, Huber W, Anders S. Moderated estimation of fold change and dispersion for RNA-seq data with DESeq2. Genome Biol. 2014;15:550. 
41. Rinker DC, Zhou X, Pitts RJ, Jones PL, Rokas A, Zwiebel LJ. RNAseq in the mosquito maxillary palp: a little antennal RNA goes a long way. bioRxiv. 2015. doi:10.1101/016998

42. Kriventseva EV, Tegenfeldt F, Petty TJ, Waterhouse RM, Simao FA, Pozdnyakov IA, et al. OrthoDB v8: update of the hierarchical catalog of orthologs and the underlying free software. Nucleic Acids Res. 2015;43: D250-6.

43. Timoshevskiy VA, Kinney NA, Debruyn BS, Mao C, Tu Z, Severson DW, et al. Genomic composition and evolution of Aedes aegypti chromosomes revealed by the analysis of physically mapped supercontigs. BMC Biol. 2014;12:27.

44. Liu L, Li Y, Wang R, Yin C, Dong Q, Hing H, et al. Drosophila hygrosensation requires the TRP channels water witch and nanchung. Nature. 2007;450:294-8.

45. Li B, Ruotti V, Stewart RM, Thomson JA, Dewey CN. RNA-Seq gene expression estimation with read mapping uncertainty. Bioinformatics. 2010; 26:493-500.

46. Jones AK, Grauso M, Sattelle DB. The nicotinic acetylcholine receptor gene family of the malaria mosquito, Anopheles gambiae. Genomics. 2005:85:176-87.

47. Pitts RJ, Derryberry Jr SL, Pulous FE, Zwiebel L. Antennal-expressed ammonium transporters in the malaria vector mosquito Anopheles gambiae. PLoS One. 2014;9:e111858.

48. Menuz K, Larter NK, Park J, Carlson JR. An RNA-seq screen of the Drosophila antenna identifies a transporter necessary for ammonia detection. PLoS Genet. 2014;10:e1004810

49. Geier M, Bosch OJ, Boeckh J. Ammonia as an attractive component of host odour for the yellow fever mosquito, Aedes aegypti. Chem Senses. 1999;24:647-53.

50. Braks MAH, Meijerink J, Takken W. The response of the malaria mosquito, Anopheles gambiae, to two components of human sweat, ammonia and Ilactic acid, in an olfactometer. Physiol Entomol. 2001;26:142-8.

51. Smallegange RC, Qiu YT, van Loon JJ, Takken W. Synergism between ammonia, lactic acid and carboxylic acids as kairomones in the host-seeking behaviour of the malaria mosquito Anopheles gambiae sensu stricto (Diptera: Culicidae). Chem Senses. 2005;30:145-52.

52. Azanchi R, Kaun KR, Heberlein U. Competing dopamine neurons drive oviposition choice for ethanol in Drosophila. Proc Natl Acad Sci U S A. 2013;110:21153-8

53. Cunningham CB, Douthit MK, Moore AJ. Octopaminergic gene expression and flexible social behaviour in the subsocial burying beetle Nicrophorus vespilloides. Insect Mol Biol. 2014;23(3):391-404.

54. Monastirioti M. Biogenic amine systems in the fruit fly Drosophila melanogaster. Microsc Res Tech. 1999;45:106-21.

55. Perry CJ, Barron AB. Neural mechanisms of reward in insects. Annu Rev Entomol. 2013:58:543-62

56. Fukumitsu $Y$, Irie $K$, Satho $T$, Aonuma $H$, Dieng $H$, Ahmad AH, et al. Elevation of dopamine level reduces host-seeking activity in the adult female mosquito Aedes albopictus. Parasit Vectors. 2012;5:92.

57. Siju KP, Hansson BS, Ignell R. Immunocytochemical localization of serotonin in the central and peripheral chemosensory system of mosquitoes. Arthropod Struct Dev. 2008;37:248-59.

58. Moffett SB, Moffett DF. Comparison of immunoreactivity to serotonin, FMRFamide and SCPb in the gut and visceral nervous system of larvae, pupae and adults of the yellow fever mosquito Aedes aegypti. J Insect Sci. 2005:5:20.

59. Gasque G, Conway S, Huang J, Rao Y, Vosshall LB. Small molecule drug screening in Drosophila identifies the 5HT2A receptor as a feeding modulation target. Sci Rep. 2013;3:srep02120.

60. Nässel DR, Winther AM. Drosophila neuropeptides in regulation of physiology and behavior. Prog Neurobiol. 2010;92:42-104.

61. Riehle MA, Garczynski SF, Crim JW, Hill CA, Brown MR. Neuropeptides and peptide hormones in Anopheles gambiae. Science. 2002;298:172-5.

62. Vogel KJ, Brown MR, Strand MR. Phylogenetic investigation of Peptide hormone and growth factor receptors in five dipteran genomes. Front Endocrinol (Lausanne). 2013;4:193.

63. Caers J, Verlinden H, Zels S, Vandersmissen HP, Vuerinckx K, Schoofs L. More than two decades of research on insect neuropeptide GPCRs: an overview. Front Endocrinol (Lausanne). 2012:3:151.

64. Hewes RS, Taghert PH. Neuropeptides and neuropeptide receptors in the Drosophila melanogaster genome. Genome Res. 2001;11:1126-42.

65. Siju KP, Reifenrath A, Scheiblich H, Neupert S, Predel R, Hansson BS, et al Neuropeptides in the antennal lobe of the yellow fever mosquito, Aedes aegypti. J Comp Neurol. 2014;522:592-608.
66. Robertson HM, Warr CG, Carlson JR. Molecular evolution of the insect chemoreceptor gene superfamily in Drosophila melanogaster. Proc Nat Acad Sci U S A. 2003;100 Suppl 2:14537-42.

67. Kwon HW, Lu T, Rutzler M, Zwiebel LJ. Olfactory responses in a gustatory organ of the malaria vector mosquito Anopheles gambiae. Proc Natl Acad Sci U S A. 2006;103:13526-31

68. Jung JW, Baeck SJ, Perumalsamy H, Hansson BS, Ahn YJ, Kwon HW. A novel olfactory pathway is essential for fast and efficient blood-feeding in mosquitoes. Sci Rep. 2015;5:13444.

69. McKenzie SK, Oxley PR, Kronauer DJ. Comparative genomics and transcriptomics in ants provide new insights into the evolution and function of odorant binding and chemosensory proteins. BMC Genomics. 2014;15:718.

70. Göpfert MC, Briegel H, Robert D. Mosquito hearing: sound-induced antennal vibrations in male and female Aedes aegypti. J Exp Biol. 1999;202:2727-38.

71. Rytz R, Croset V, Benton R. lonotropic receptors (IRs): chemosensory ionotropic glutamate receptors in Drosophila and beyond. Insect Biochem Mol Biol. 2013;43:888-97.

72. Croset V, Rytz R, Cummins SF, Budd A, Brawand D, Kaessmann H, et al. Ancient protostome origin of chemosensory ionotropic glutamate receptors and the evolution of insect taste and olfaction. PLoS Genet. 2010;6: e1001064

73. Benton R. Chemical sensing in Drosophila. Curr Opin Neurobiol. 2008;18: 357-63.

74. Ni L, Bronk P, Chang EC, Lowell AM, Flam JO, Panzano VC, et al. A gustatory receptor paralogue controls rapid warmth avoidance in Drosophila. Nature. 2013;500:580-4

75. Xiang Y, Yuan Q, Vogt N, Looger LL, Jan LY, Jan YN. Light-avoidancemediating photoreceptors tile the Drosophila larval body wall. Nature. 2010; 468:921-6.

76. Kent LB, Walden KK, Robertson HM. The Gr family of candidate gustatory and olfactory receptors in the yellow-fever mosquito Aedes aegypti. Chem Senses. 2008;33:79-93.

77. Zelle KM, Lu B, Pyfrom SC, Ben-Shahar Y. The genetic architecture of degenerin/epithelial sodium channels in Drosophila. G3 (Bethesda). 2013;3: 441-50.

78. Fowler MA, Montell C. Drosophila TRP channels and animal behavior. Life Sci. 2013:92:394-403.

79. Hall AB, Basu S, Jiang X, Qi Y, Timoshevskiy VA, Biedler JK, et al. A male-determining factor in the mosquito Aedes aegypti. Science. 2015; 348:1268-70.

80. Hall AB, Timoshevskiy VA, Sharakhova MV, Jiang X, Basu S, Anderson MA, et al. Insights into the preservation of the homomorphic sex-determining chromosome of Aedes aegypti from the discovery of a male-biased gene tightly linked to the M-locus. Genome Biol Evol. 2014;6:179-91.

81. Fox AN, Pitts RJ, Robertson HM, Carlson JR, Zwiebel LJ. Candidate odorant receptors from the malaria vector mosquito Anopheles gambiae and evidence of down-regulation in response to blood feeding. Proc Natl Acad Sci U S A. 2001;98:14693-7.

82. Qiu YT, Gort G, Torricelli R, Takken W, van Loon JJ. Effects of blood-feeding on olfactory sensitivity of the malaria mosquito Anopheles gambiae: application of mixed linear models to account for repeated measurements. J Insect Physiol. 2013;59:1111-8.

83. Siju KP, Hill SR, Hansson BS, Ignell R. Influence of blood meal on the responsiveness of olfactory receptor neurons in antennal sensilla trichodea of the yellow fever mosquito, Aedes aegypti. J Insect Physiol. 2010;56:659-65.

84. Akbari OS, Papathanos PA, Sandler JE, Kennedy K, Hay BA. Identification of germline transcriptional regulatory elements in Aedes aegypti. Sci Rep. 2014;4:3954.

85. Bohbot JD, Dickens JC. Characterization of an enantioselective odorant receptor in the yellow fever mosquito Aedes aegypti. PLoS One. 2009:4:e7032

86. Trapnell C, Hendrickson DG, Sauvageau M, Goff L, Rinn JL, Pachter L. Differential analysis of gene regulation at transcript resolution with RNA-seq Nat Biotechnol. 2013;31:46-53.

87. Haas BJ, Papanicolaou A, Yassour M, Grabherr M, Blood PD, Bowden J, et al. De novo transcript sequence reconstruction from RNA-seq using the Trinity platform for reference generation and analysis. Nat Protoc. 2013;8:1494-512.

88. Fu L, Niu B, Zhu Z, Wu S, Li W. CD-HIT: accelerated for clustering the nextgeneration sequencing data. Bioinformatics. 2012;28:3150-2. 
89. Li W, Godzik A. CD-HIT: a fast program for clustering and comparing large sets of protein or nucleotide sequences. Bioinformatics. 2006;22:1658-9.

90. Standage DS, Brendel VP. ParsEval: parallel comparison and analysis of gene structure annotations. BMC Bioinformatics. 2012;13:187.

91. Haas BJ, Delcher AL, Mount SM, Wortman JR, Smith Jr RK, Hannick LI, et al. Improving the Arabidopsis genome annotation using maximal transcript alignment assemblies. Nucleic Acids Res. 2003;31:5654-66.

Submit your next manuscript to BioMed Central and we will help you at every step:

- We accept pre-submission inquiries

- Our selector tool helps you to find the most relevant journal

- We provide round the clock customer support

- Convenient online submission

- Thorough peer review

- Inclusion in PubMed and all major indexing services

- Maximum visibility for your research 